\title{
Testing the Conditional Mean Function of Autoregressive Conditional Duration Models
}

Hautsch, Nikolaus

Publication date:

2006

Document version

Publisher's PDF, also known as Version of record

Citation for published version (APA):

Hautsch, N. (2006). Testing the Conditional Mean Function of Autoregressive Conditional Duration Models.

Department of Economics, University of Copenhagen. 


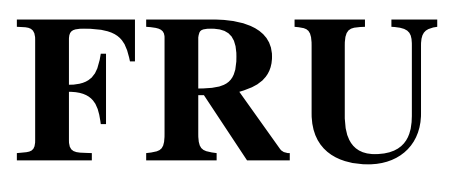

Finance Research Unit

\title{
Testing the Conditional Mean Function of Autoregressive Conditional Duration Models
}

\author{
Nikolaus Hautsch
}

No. $2006 / 06$

Finance Research Unit

Department of Economics

University of Copenhagen

http://www.econ.ku.dk/FRU 


\title{
TESTING THE CONDITIONAL MEAN FUNCTION OF AUTOREGRESSIVE CONDITIONAL DURATION MODELS
}

\author{
NIKOLAUS HAUTSCH* \\ DEPARTMENT OF ECONOMICS \\ UNIVERSITY OF COPENHAGEN
}

\begin{abstract}
In this paper, we suggest and evaluate specification tests to test the validity of the conditional mean function implied by Autoregressive Conditional Duration (ACD) models. We propose Lagrange multiplier tests against sign bias alternatives, various types of conditional moment tests and integrated conditional moment tests which are consistent against all possible alternatives. In a Monte-Carlo study we investigate the finite sample properties of the individual tests. Moreover, the testing framework is applied to a variety of existing and new ACD specifications using financial duration data based on NYSE trading. We show that conditional moment tests have the highest power to detect general types of misspecifications. Moreover, we provide evidence that most ACD specifications are too simple and are clearly rejected. It turns out that flexible parameterizations of the news impact function are necessary to appropriately model financial durations. A semiparametric ACD model proposed in this paper seem to be a valuable alternative to existing approaches.
\end{abstract}

\section{INTRODUCTION}

The seminal papers of Engle and Russell (1998) and Engle (2000) have been the starting point for a wide range of studies focussing on the specification and application of autoregressive conditional duration (ACD) models. Not surprisingly, this string of the literature is strongly affected by its resemblance to the GARCH framework. Bauwens and Giot (2000), for example, introduce the Log-ACD model which is the counterpart to the Log-GARCH model. Dufour and Engle (2000) and Hautsch (2003) suggest different types of Box-Cox-ACD models whereas Fernandes and Grammig (2006) build a family of asymmetric ACD models on the basis of the framework of asymmetric GARCH processes introduced by Hentschel (1995). Moreover, regime-switching ACD specifications have been introduced by Zhang, Russell, and Tsay (2001) and recently extended by Meitz

December 13, 2006.

JEL Classifications: C22, C41, C52.

Keywords: Augmented ACD models, semiparametric ACD models, news impact function, Lagrange multiplier tests, (integrated) conditional moment tests

* Address: Studiestraede 6, DK-1455 Copenhagen K, Denmark

tel: ++ 45353 23022, fax: ++ 45353 23000, email: nikolaus.hautsch@econ.ku.dk.

Early versions of this paper have been presented at the European meeting of the Econometric Society in Venice, 2002, the annual meeting of the German Economic Association in Innsbruck, 2002, and the EC $^{2}$ conference in Bologna, 2002. Helpful comments by Luc Bauwens, Pierre Giot, Michael Lechner, David Veredas, Winfried Pohlmeier, Timo Teräsvirta, and the seminar participants at the University of Konstanz, the University of St. Gallen, the Université Catholique de Louvain, the University of Sydney as well as the University of Technology, Sydney, are gratefully acknowledged. The work was supported in part by the European Community's Human Potential Programme under contract HPRN-CT-2002-00232, [MICFINMA]. All remaining errors are my sole responsibility. 
and Teräsvirta (2006) to allow for smooth transition specifications. Finally, Jasiak (1998) introduces a fractionally integrated ACD model.

The fundamental building block of the ACD model is the specification of the conditional expected duration mean. The validity of this conditional mean restriction is the basic assumption underlying the ACD model and is the prerequisite to ensure the quasi maximum likelihood (QML) properties of the Exponential ACD (EACD) specification (see Engle and Russell, 1998). Nonetheless, most diagnostics that have been applied in recent ACD literature are not appropriate for an explicit test of the conditional mean restriction. A common way to evaluate the ACD model is to check the goodness-of-fit, for example based on residual diagnostics, in-sample density forecast evaluations (see Bauwens et al, 2004) or based on nonparametric specification tests against distributional misspecification (see Fernandes and Grammig, 2005). However, these tests do not allow to identify whether a possible rejection is due to a violation of distributional assumptions or due to a misspecification of the first conditional moment implied by an ACD process. Therefore, the fundamental motivation for this study is the need for diagnostic tests which are particularly sensitive against violations of the conditional mean restriction but not necessarily against misspecifications of higher order moments.

The contribution of this paper is two-fold. First, we suggest and evaluate a wide range of different types of conditional moment tests for ACD models on the basis of an extensive Monte-Carlo study. Second, we apply these tests to various types of ACD models and illustrate the need for specifications implying a flexible parameterization of the news impact function. In this context, we suggest two new types of flexible ACD models and demonstrate their usefulness in light of the proposed testing framework.

A natural way to construct a specification test that allows to exclusively test the conditional mean restriction is to rely on the QML property of the EACD model and to apply the framework of conditional moment tests. In this context, we consider several types of specification tests:(i) As proposed by Engle and Russell (1994) and extended by Meitz and Teräsvirta (2006) one simple proceeding is to specify Lagrange Multiplier (LM) tests which have optimal power against local alternatives. However, while Meitz and Teräsvirta construct LM tests against particular parametric alternatives, our focus is on LM tests which allow to test for misspecifications of more general and unknown form. Therefore, following Engle and Ng (1993), we propose LM tests against sign bias alternatives and nonlinearities in the news impact function. Such tests allow to test whether the functional form of the conditional mean function is appropriate to account for possibly nonlinear news response effects. (ii) In order to apply a more general test against violations of the conditional mean restriction, we use conditional moment (CM) tests as introduced by Newey (1985). These tests are consistent against a finite number of possible alternatives since they rely on a finite number of conditional moment restrictions. In this context, we 
evaluate a wide range of different $\mathrm{CM}$ tests based on various weighting functions. (iii) Moreover, we suggest the use of integrated conditional moment (ICM) tests proposed by Bierens (1982 and 1990). By employing an infinite number of conditional moments this test possesses the property of consistency against all possible alternatives and thus is a generalization of the CM test. de Jong (1996) shows how to generalize Bierens' test towards data dependence and develops a simulation procedure that is capable of establishing critical values that are asymptotically valid. We illustrate how to adapt this test to the class of ACD models.

This testing framework is applied to three major types of ACD models. First, we propose a new type of augmented ACD model which includes additive as well as multiplicative stochastic components based on a flexible parameterization of the news impact function. This specification nests the Hentschel (1995) type ACD model introduced by Fernandes and Grammig (2006) as well as a variety of nonlinear ACD specifications. Second, we propose an ACD specification based on a semiparametric specification of the news impact function in the spirit of Engle and $\mathrm{Ng}$ (1993). Here, the news impact is modelled in terms of a piecewise linear spline function. Third, we apply the class of Threshold ACD (TACD) models introduced by Zhang, Russell, and Tsay (2001).

The different types of ACD models are used as data generating processes in a Monte Carlo study where we analyze the size and power properties of the different tests. It is shown that CM tests have the highest power and seem to be very useful diagnostic tools for the evaluation of ACD models. Clearly less power is observed for LM tests and ICM tests. However, we also find evidence for size distortions in finite samples. Finally, the proposed models and tests are applied to financial durations from the AOL, Coca-Cola, Disney and GE stock traded at the New York Stock Exchange (NYSE). We focus on trade durations that play an important role in market microstructure analysis ${ }^{1}$ and on price durations that are strongly related to volatility measures ${ }^{2}$. We provide strong evidence for nonlinearities in the news response function. It is shown that more simple ACD specifications are rejected for nearly all duration series under consideration. Clear improvements of the goodness-offit are obtained by more flexible and higher parameterized ACD models. The best results are obtained for augmented ACD models that explicitly account for nonlinearities in the news response as well as for specifications based on a semiparametric news impact function. Particularly the latter seem to be a valuable flexible alternative to existing approaches. Nevertheless, especially for price durations it turns out that even high flexible ACD models still reveal problems to ensure the conditional mean restriction.

The rest of the paper is organized in the following way: In Section 2, we present different types of ACD specifications. Section 3 introduces model specification tests based on LM

\footnotetext{
${ }^{1}$ See, for example, Easley and O‘Hara (1992), Diamond and Verrecchia (1987) or Admati and Pfleiderer (1988).

${ }^{2}$ See Engle and Russell (1998), Giot (2000) or Gerhard and Hautsch (2002).
} 
tests, CM tests and ICM tests. Section 4 presents an extensive Monte Carlo study which allows us to gain deeper insights into the power and the size of the particular tests. In Section 5, we show empirical results based on an application of the proposed framework to financial durations generated from the trading process at the NYSE. The conclusions are given in Section 6 .

\section{THE ACD MODEL}

Let $x_{i}=\vartheta_{i}-\vartheta_{i-1}$ denote the time between two events occurring at time $\vartheta_{i}$ and $\vartheta_{i-1}$, respectively. Engle and Russell (1998) propose to specify the duration process based on a dynamic parameterization of the conditional mean function $\Psi_{i} \equiv \mathrm{E}\left[x_{i} \mid \mathcal{I}_{i-1} ; \theta_{0}\right]$, where $\mathcal{I}_{i-1}$ denotes the filtration up to period $i-1$ and $\theta_{0}$ is a parameter vector $\theta_{0} \in \Theta$ with $\Theta$ some compact subset of $\mathbb{R}^{k}$. By defining $\varepsilon_{i}$ as an i.i.d. random variable with positive support and $\mathrm{E}\left[\varepsilon_{i}\right]=1$, the ACD model is given by

$$
x_{i}=\Psi_{i} \varepsilon_{i} .
$$

Hence, the specification of an ACD model includes (i) the choice of the functional form for the conditional mean function $\Psi_{i}$ and (ii) the choice of an appropriate distribution for $\varepsilon_{i}$. The latter issue is crucial when one is interested not only in predictions of the duration mean but also in forecasts of quantiles of the complete conditional distribution. However, in this paper, we focus exclusively on predictions of the duration mean and rely on the QML property of the EACD model. Engle (2000) shows that the results of Bollerslev and Wooldridge (1992) concerning the QML property of the $\operatorname{GARCH}(p, q)$ model can be carried over to the $\operatorname{EACD}(p, q)$ model. Therefore, the maximization of the quasi log likelihood function implied by the EACD model leads to consistent estimates even when the true density function is not exponential.

The most simple ACD model and direct counterpart to the (linear) GARCH model has been proposed by Engle and Russell (1998) in their seminal paper and is given by ${ }^{3}$

$$
\begin{aligned}
\Psi_{i} & =\omega+\alpha x_{i-1}+\beta \Psi_{i-1} \\
& =\omega+\alpha \Psi_{i-1} \varepsilon_{i-1}+\beta \Psi_{i-1},
\end{aligned}
$$

where $\omega$ denotes a constant, $\alpha$ is the innovation parameter, and $\beta$ denotes the persistence parameter. This model implies a linear news impact curve, i.e. a linear relationship between the past innovation $\varepsilon_{i-1}$ and the current expected conditional mean $\Psi_{i}$. The slope of the news impact curve is given by $\alpha \Psi_{i-1}$, thus this specification is based on a multiplicative stochastic component.

\footnotetext{
${ }^{3}$ For simplicity of exposition the following considerations are restricted to models with a lag order of one.
} 
Here, we propose a new type of augmented ACD model which nests a wide range of specifications and is given by

(3) $\Psi_{i}^{\delta_{1}}=\omega+\alpha \Psi_{i-1}^{\delta_{1}}\left(\left|\varepsilon_{i-1}-b\right|+c\left(\varepsilon_{i-1}-b\right)\right)^{\delta_{2}}+\nu\left(\left|\varepsilon_{i-1}-b\right|+c\left(\varepsilon_{i-1}-b\right)\right)^{\delta_{2}}+\beta \Psi_{i-1}^{\delta_{1}}$,

where $\delta_{1}, \delta_{2} \geq 0$. We call this specification augmented ACD (AGACD) model. It includes both a multiplicative as well as additive stochastic component. For $\nu=0$, the additive stochastic component is not existing and the AGACD model corresponds to an ACD specification proposed by Fernandes and Grammig (2006) which is the counterpart to the augmented GARCH process proposed by Hentschel (1995). ${ }^{4}$ Correspondingly, for $\alpha=0$, the model contains only an additive stochastic component. The model allows for a news impact curve which is kinked at $b$. The parameter $\delta_{2}$ determines the shape of the piecewise functions around the kink. For $\delta_{2}>1$ the shape is convex while for $\delta_{2}<1$ it is concave. Finally, $\delta_{1}$ drives a possible power transformation of $\Psi_{i}$. For $\delta_{1} \rightarrow 0$, the model is based on a logarithmic transformation of $\Psi_{i}$. In this case, the multiplicative and additive components of the model coincide. The AGACD model encompasses several important special cases:

(i) For $\alpha=0, \delta_{1} \rightarrow 0$, and $c=1$ it nests the so-called EXponential ACD model proposed by Dufour and Engle $(2000)^{5}$ and given by

$$
\ln \Psi_{i}=\omega+\alpha \varepsilon_{i-1}+c\left|\varepsilon_{i-1}-1\right|+\beta \ln \Psi_{i-1} .
$$

It captures features of the EGARCH specification proposed by Nelson (1991) and allows for a linear news impact function which is kinked at $\varepsilon_{i-1}=1$.

(ii) For $\alpha=0, b=0$, and $c=0$ it nests the so-called Box-Cox ACD (BACD) model introduced by Hautsch (2003) which is based on power transformations of $\Psi_{i}$ and $\varepsilon_{i}$ and is given by

$$
\Psi_{i}^{\delta_{1}}=\omega+\alpha \varepsilon_{i-1}^{\delta_{2}}+\beta \Psi_{i-1}^{\delta_{1}} .
$$

It is easily illustrated that it can be re-written in terms of Box-Cox transformations. ${ }^{6}$ This specification allows for concave, convex as well as linear news impact curves. For $\delta_{1} \rightarrow 0, \delta_{2} \rightarrow 0$, it simplifies to the Log-ACD (LACD) model proposed by Bauwens and Giot (2000) and is given by

$$
\begin{aligned}
\ln \Psi_{i} & =\omega+\alpha \ln \varepsilon_{i-1}+\beta \ln \Psi_{i-1} \\
& =\omega+\alpha \ln x_{i-1}+(\beta-\alpha) \ln \Psi_{i-1} .
\end{aligned}
$$

(iii) For $\delta_{1}=\delta_{2}=1, b=c=0$, it nests a specification which we call Additive and Multiplicative ACD (AMACD) model and is based on a (linear) additive and multiplicative

\footnotetext{
${ }^{4}$ Though Fernandes and Grammig call their specification augmented ACD model as well, here, however, we call it $\mathrm{H}$ (entschel)-ACD model in order to avoid confusion.

${ }^{5}$ They use this notation in order to prevent a confusion with the Exponential ACD (EACD) model based on an exponential distribution.

${ }^{6}$ See Hautsch (2003).
} 
innovation component. It is given by

$$
\Psi_{i}=\omega+\left(\alpha \Psi_{i-1}+\nu\right) \varepsilon_{i-1}+\beta \Psi_{i-1} .
$$

For $\nu=0$ it corresponds to the basic linear ACD specification.

Furthermore, we propose a new ACD specification on the basis of a semiparametric news response function. Following Engle and Ng (1993) we specify an ACD model based on a news impact curve which is parameterized as a linear spline function with knots at given break points of $\varepsilon_{i-1}$. In particular, the range of $\varepsilon_{i-1}$ is divided into $m$ intervals where $m^{-}\left(m^{+}\right)$denotes the number of intervals in the range $\varepsilon_{i-1}<1\left(\varepsilon_{i-1}>1\right)$ with $m=m^{-}+m^{+}$. We denote the breakpoints by $\left\{\bar{\varepsilon}_{m^{-}}, \ldots, \bar{\varepsilon}_{-1}, \bar{\varepsilon}_{0}, \bar{\varepsilon}_{1}, \ldots, \bar{\varepsilon}_{m^{+}}\right\}$. Note that the particular intervals have not to be equally sized, nor we need the same number of intervals on each side of $\bar{\varepsilon}_{0}$. The so-called Semiparametric ACD (SPACD) model is given by

(8) $\ln \Psi_{i}=\omega+\sum_{j=0}^{m^{+}} \alpha_{j}^{+} \mathbf{1}_{\left\{\varepsilon_{i-1} \geq \bar{\varepsilon}_{j}\right\}}\left(\varepsilon_{i-1}-\bar{\varepsilon}_{j}\right)+\sum_{j=0}^{m^{-}} \alpha_{j}^{-} \mathbf{1}_{\left\{\varepsilon_{i-1}<\bar{\varepsilon}_{j}\right\}}\left(\varepsilon_{i-1}-\bar{\varepsilon}_{j}\right)+\beta \ln \Psi_{i-1}$.

As pointed out by Engle and Ng (1993), a slow increase of $m$ as a function of the sample size should asymptotically give a consistent estimate of the news impact curve. This specification allows for quite flexible (nonlinear) news responses but does not necessarily nest the family of AGACD models.

The theoretical properties of the AGACD and SPACD model can be derived by expressing them in the form of a generalized polynomial random coefficient autoregressive model as introduced by Carrasco and Chen (2002) for the analysis of GARCH processes and applied to ACD type models by Fernandes and Grammig (2006). By constituting a general class of ACD models in the form

$$
\zeta_{i}=\mathcal{A}\left(\varepsilon_{i}\right) \zeta_{i-1}+\mathcal{B}\left(\varepsilon_{i}\right)
$$

and parameterizing $\mathcal{A}(\cdot)$ and $\mathcal{B}(\cdot)$ appropriately, we can encompasses all ACD specifications discussed above. The theoretical properties for this class of models are derived by Fernandes and Grammig (2006). In particular, they refer to the general results of Carrasco and Chen (2002) and Mokkadem (1990) which provide sufficient conditions to ensure $\beta$-mixing, strict stationarity and the existence of higher order moments for the class of generalized polynomial random coefficient autoregressive models. As shown by Fernandes and Grammig (2006), the establishment of stationarity conditions for the particular ACD specifications requires to impose restrictions on the functions $\mathcal{A}(\cdot)$ and $\mathcal{B}(\cdot) .^{7}$

A further type of ACD model arises from the class of threshold models. Zhang, Russell, and Tsay (2001) introduce a threshold ACD model which allows the expected duration to depend nonlinearly on past information variables. The TACD model can be seen

\footnotetext{
${ }^{7}$ See Proposition 1 in Fernandes and Grammig (2006) as well as Proposition 2 and 4 in Carrasco and Chen (2002), 2002.
} 
as a generalization of the threshold GARCH models introduced by Rabemananjara and Zakoian (1993) and Zakoian (1994). By categorizing the durations into $K$ categories with thresholds $\bar{x}_{1}, \bar{x}_{2}, \ldots, x_{K-1}$, a $K$-regime $\operatorname{TACD}(1,1)$ model is given by

$$
\left\{\begin{array}{l}
x_{i}=\Psi_{i} \varepsilon_{i} \\
\Psi_{i}=\omega^{(k)}+\alpha^{(k)} x_{i-j}+\beta^{(k)} \Psi_{i-j},
\end{array} \quad \text { if } x_{i-1} \in\left(\bar{x}_{k-1}, \bar{x}_{k}\right]\right.
$$

where $\omega^{(k)}>0, \alpha^{(k)} \geq 0$ and $\beta^{(k)} \geq 0$ are regime-switching ACD parameters. Hence, whereas in the SPACD model only the impact of lagged innovations is regime-dependent, in the TACD model also the persistence terms are allowed to be regime-switching. Because of the latter, the derivation of theoretical properties is not straightforward. Zhang, Russell, and Tsay (2001) derive conditions for geometric ergodicity and the existence of moments for the $\operatorname{TACD}(1,1)$ case. Extensions of this framework to the case of smooth transitions have been recently introduced by Meitz and Teräsvirta (2006). In order to restrict the extent of the empirical study in this paper, we do not discuss these models here.

\section{SpeCification tests For ACD MODELS}

The null hypothesis of correct specification of the conditional mean function of the ACD model can be formulated in terms of the ACD residuals $e_{i} \equiv x_{i} / \hat{\Psi}_{i}$,

$$
H_{0}: \quad \operatorname{Pr}\left[\mathrm{E}\left[e_{i}-1 \mid \mathcal{I}_{i-1}\right]=0\right]=1,
$$

or, alternatively, in terms of the martingale differences $x_{i}-\hat{\Psi}_{i}$, i.e.

$$
H_{0}: \quad \operatorname{Pr}\left[\mathrm{E}\left[x_{i}-\hat{\Psi}_{i} \mid \mathcal{I}_{i-1}\right]=0\right]=1 .
$$

Asymptotically, this distinction should make no difference, however, in finite samples it can be an issue. Correspondingly, the alternative hypotheses are formulated as

$$
H_{1}: \operatorname{Pr}\left[\mathrm{E}\left[e_{i}-1 \mid \mathcal{I}_{i-1}\right]=0\right]<1 \quad \text { or } \quad H_{1}: \operatorname{Pr}\left[\mathrm{E}\left[x_{i}-\hat{\Psi}_{i} \mid \mathcal{I}_{i-1}\right]=0\right]<1 .
$$

In the following we discuss different types of LM tests, CM tests based on a finite number of conditional moment restrictions and, ICM tests based on an infinite number of conditional mean restrictions as omnibus tests against any form of misspecification.

3.1. Lagrange Multiplier (LM) Tests. In econometric literature, the LM test has proven to be a useful diagnostic tool to detect model misspecifications. See for example, Breusch (1978), Breusch and Pagan (1979, 1980), Godfrey (1978a, 1978b), or for an overview in Engle (1984). In the following we restrict our consideration to the case of QML estimation of the ACD model, i.e. we maximize the quasi likelihood function

$$
\mathcal{L}_{Q M L}(\theta)=-\sum_{i=1}^{n}\left[\ln \Psi_{i}+\frac{x_{i}}{\Psi_{i}}\right]=-\sum_{i=1}^{n} l_{i} .
$$

In this case the LM test statistic is computed as

$$
\Upsilon_{L M}=f_{0}^{\prime} z_{0}\left(z_{0}^{\prime} z_{0}\right)^{-1} z_{0}^{\prime} f_{0},
$$


where

$$
f_{0}=\left(\frac{x_{1}}{\Psi_{1}}-1, \ldots, \frac{x_{n}}{\Psi_{n}}-1\right)^{\prime}, \quad z_{0}=\left(\frac{1}{\Psi_{1}} \frac{\partial \Psi_{1}}{\partial \theta_{0}}, \ldots, \frac{1}{\Psi_{n}} \frac{\partial \Psi_{n}}{\partial \theta_{0}}\right)^{\prime}
$$

both evaluated under the null. It is easy to show that this test statistic corresponds to the uncentered $R^{2}$ from a regression of $f_{0}$ on $z_{0}$ and is commonly computed as $n R^{2}$ where $R^{2}$ is the uncentered $R^{2}$ from a regression of a vector of ones on the scores of the model. To perform the LM test it is necessary to specify a general model which encompasses the model under the null. Meitz and Teräsvirta (2006) discuss different LM tests agains particular parametric alternatives. However, here we focus on a more general form of LM test which allows to test for misspecifications of the conditional mean function of unknown form. Assume that the ACD specification under the null is a special case of a more general (additive) model of the form

$$
\Psi_{i}=\Psi_{i}^{0}+\theta_{a}^{\prime} z_{a i},
$$

where $\Psi_{i}^{0}$ denotes the conditional mean function under the null depending on the parameter vector $\theta_{0}$, while $\theta_{a}$ and $z_{a i}$ denote the vectors of additional parameters and missing variables, respectively. Thus we can test for the correct specification of the null model by testing the parameter restriction $\theta_{a}=0$. Following the idea of Engle and $\mathrm{Ng}$ (1993) we specify $z_{a i}$ in terms of so-called sign bias variables $\mathbf{I}_{\left\{\varepsilon_{i-1}<1\right\}}, \mathbf{I}_{\left\{\varepsilon_{i-1}<1\right\}} \varepsilon_{i-1}$ and $\mathbf{I}_{\left\{\varepsilon_{i-1} \geq 1\right\}} \varepsilon_{i-1}$, and extensions thereof. As explained in more details in Section 4, we employ different versions of this test which allows to investigate whether the specification is appropriate to capture possible nonlinearities in the news impact function. The resulting LM test is formulated based on the auxiliary regression

$$
e_{i}=z_{0 i}^{\prime} \tilde{\beta}_{0}+z_{a i}^{\prime} \tilde{\beta}_{a}+u_{i}
$$

where $u_{i}$ is a zero mean i.i.d. error term, $\tilde{\beta}_{0}$ and $\tilde{\beta}_{a}$ are regression coefficients, $z_{0 i}=$ $1 / \Psi_{i}^{0} \cdot \partial \Psi_{i}^{0} / \partial \theta_{0}$ and $z_{a i}=1 / \Psi_{i}^{0} \cdot \partial \Psi_{i}^{0} / \partial \theta_{a}$ evaluated at $\theta_{a}=0$ and at the QML estimator under the null. Then, the statistic is given by $n$ times the $R^{2}$ from the regression (16) and follows asymptotically a $\chi^{2}(m)$ distribution where $m$ denotes the number of restrictions. However, as discussed in Meitz and Teräsvirta (2006), this test is not robust if the ACD errors $\varepsilon_{i}$ are not exponentially distributed. They suggest to follow the approach by Wooldridge (1990) and to apply the following procedure: (i) Compute the residuals, $r_{i}$, from a regression of $z_{a i}$ on $z_{0 i}$. (ii) Regress a vector of ones on $r_{i}\left(x_{i} / \Psi_{i}-1\right)$ and compute the sum of squared residuals, SSR. (iii) Compute the asymptotically $\chi^{2}(m)$ distributed test statistic as $n$ times SSR. As illustrated by Wooldridge (1990) this procedure leads to a consistent test which is (asymptotically) not affected by violations of the underlying distributional assumptions.

3.2. Conditional Moment (CM) Tests. The main idea behind the CM test is to test the validity of conditional moment restrictions implied by the data which should hold 
when the model is correctly specified. In the ACD framework the conditional moment test is based on a conditional moment function $r\left(x_{i}, \theta_{0}\right)$ which should hold conditionally on the past filtration, thus $\mathrm{E}\left[r\left(x_{i}, \theta_{0}\right) \mid \mathcal{I}_{i-1}\right]=0$. Newey (1985) proposes to build a $\mathrm{CM}$ test based on the unconditional moment restrictions of the form

$$
m_{j}\left(x_{i}, \theta_{0}\right)=r\left(x_{i}, \theta_{0}\right) w_{j}\left(\mathcal{I}_{i-1}, \theta_{0}\right),
$$

where $w_{j}\left(\mathcal{I}_{i-1}, \theta_{0}\right)$ is a weighting function that is indexed by $j$ and is based on the past filtration. Hence, $w_{j}(\cdot)$ is orthogonal to $r\left(x_{i}, \theta_{0}\right)$ and thus $\mathrm{E}\left[m_{j}(\cdot)\right]=0$. As shown by Newey, an asymptotically $\chi^{2}$ distributed test statistic is built based on the vector of unconditional moment restrictions and the vector of scores. In particular, defining $g\left(x, \theta_{0}\right)$ as a vector that includes the vector of the unconditional moment restrictions $m\left(x, \theta_{0}\right)=$ $\left(m_{1}(\cdot), \ldots, m_{j}(\cdot), \ldots\right)^{\prime}$ and the score vector $s\left(x, \theta_{0}\right)$, i.e. $g\left(x, \theta_{0}\right)=\left(m\left(x, \theta_{0}\right), s\left(x, \theta_{0}\right)\right)^{\prime}$, the $\mathrm{CM}$ test statistic is given by

$$
\Upsilon_{C M}=e_{1} g\left(x, \theta_{0}\right)\left[g\left(x, \theta_{0}\right)^{\prime} g\left(x, \theta_{0}\right)\right]^{-1} g\left(x, \theta_{0}\right)^{\prime} e_{1} \stackrel{a}{\sim} \chi^{2}(m),
$$

where $e_{1}$ denotes a vector of ones.

According to the null hypotheses (11) or (12), a straightforward choice of the conditional moment restriction is

$$
r\left(x_{i}, \theta_{0}\right)=e_{i}-1
$$

or

$$
r\left(x_{i}, \theta_{0}\right)=x_{i}-\hat{\Psi}_{i} .
$$

Valuable choices of the weighting functions $w_{j}(\cdot)$ are sign bias variables (see Section 3.1) and/or functionals (e.g. moments) of past durations. ${ }^{8}$

A well known result is that the power of the CM test depends heavily on the choice of the weighting functions. For this reason Newey (1985) illustrates how to obtain an optimal conditional moment test with maximal local power. It is shown that the LM test corresponds to an optimal CM test in the case of a particular local alternative. However, since the CM test is based on a finite number of conditional moment restrictions, it cannot be consistent against all possible alternatives.

3.3. Integrated Conditional Moment (ICM) Tests. Bierens (1990) illustrates that any CM test of functional form can be converted into a chi-square test that possesses the property of consistency against all possible alternatives. The main idea behind the consistent conditional moment test is based on the following lemma:

Lemma [Lemma 1, Bierens, 1990]: Let $\varrho$ be a random variable satisfying the condition $\mathrm{E}|\varrho|<\infty$ and let $z$ be a bounded random variable in $\mathbb{R}$ with $\operatorname{Pr}[\mathrm{E}(\varrho \mid z)=0]<1$. Then

\footnotetext{
${ }^{8}$ For more details, see Section 4.
} 
the set $S=\{t \in \mathbb{R}: \mathrm{E}[\varrho \exp (t z)]=0\}$ is countable and thus has Lebesgue measure zero.

Bierens shows that $\mathrm{E}[\varrho \exp (t z)] \neq 0$ in a neighborhood of $t=t_{0}$ where $t_{0}$ is such that $\mathrm{E}\left[\varrho \exp \left(t_{0} z\right)\right]=0$ and $\operatorname{Pr}[\mathrm{E}[\varrho \exp (t z) \mid z]=0]<1$. de Jong (1996) extends Bierens' test towards the case of serial dependent data. In the following, we assume that the duration process is stationary and obeys the concept of $\nu$-stability. Moreover it is supposed that $\mathrm{E} \mid \varepsilon_{i}-$ $1 \mid<\infty$. By assuming that the model is misspecified, i.e. $\operatorname{Pr}\left[\mathrm{E}\left[r\left(x_{i}, \theta_{0}\right) \mid \mathcal{I}_{i-1}\right]=0\right]<1$ and replacing the conditioning information by $\xi\left(x_{i-1}\right), \xi\left(x_{i-2}\right), \ldots$ where $\xi(\cdot)$ is a bounded oneto-one mapping from $\mathbb{R}$ into $\mathbb{R}$, the $S=\left\{t \in \mathbb{R}^{d}: \mathrm{E}\left[r\left(x_{i}, \theta_{0}\right) \exp \left(\sum_{j=1}^{d} t_{j} \xi\left(x_{i-j}\right)\right)\right]=0\right\}$ with $d=\min (i-1, c)$ has Lebesgue measure zero. Therefore, de Jong (1996) suggests a consistent CM test based on the unconditional moment restriction

$$
\hat{M}_{n}(t)=n^{-1 / 2} \sum_{i=1}^{n} r\left(x_{i}, \theta_{0}\right) \exp \left(\sum_{j=1}^{d} t_{j} \xi\left(x_{i-j}\right)\right)
$$

where $\theta_{0}$ is estimated consistently by QML. de Jong points out that a conditional moment restriction test based on $d=c<n$ does not allow us to consistently test the hypotheses $H_{0}$ and $H_{1}$ for an infinite number of lags and thus $d=i-1$ should be preferred. Eq. (21) has the property that under the alternative hypothesis $H_{1}, \operatorname{plim}_{n \rightarrow \infty} \hat{M}_{n}(t) \neq 0$ for all $t$ except in a set with Lebesgue measure zero. Therefore, the principle of the consistent conditional moment test is to employ a class of weighting functions which are indexed by a continuous nuisance parameter (vector) $t$. Since this nuisance parameter is integrated out, this test is called integrated conditional moment (ICM) test. The lemma above implies that by choosing a vector $t^{\prime} \notin S$, a consistent CM test is obtained. However, $S$ depends on the distribution of the data, and thus it is impossible to choose a fixed vector $t$ for which the test is consistent. As suggested by de Jong (1996), a solution to this problem is to achieve test consistency by maximizing a functional of $\hat{M}_{n}(t)$ over a compact subset $\Xi$ of $\mathbb{R}^{c}$. The main idea is that a vector $t^{\prime}$ which maximizes a test statistic based on $\hat{M}_{n}(t)$ cannot belong to the set $S$. By defining a space for infinite sequences $\left\{t_{1}, t_{2}, \ldots\right\}$ as

$$
\Xi=\left\{t: a_{j} \leq t_{j} \leq b_{j} \forall j ; t_{j} \in \mathbb{R}\right\}
$$

where $a_{j}<b_{j}$ and $\left|a_{j}\right|,\left|b_{j}\right| \leq B j^{-2}$ for some constant $B$, de Jong suggests to consider the use of a functional of $\sup _{t \in \Xi}\left|\hat{M}_{n}(t)\right|$ as test statistic. Consequently, a difficulty arises by the fact that the limiting distribution of the test statistic $\sup _{t \in \Xi}\left|\hat{M}_{n}(t)\right|$ is case-dependent which prevents the use of generally applicable critical values. For this reason de Jong introduces a simulation procedure based on a conditional Monte Carlo approach. In particular, he shows that under the null, the moment restriction (21) has the same asymptotic 
finite-dimensional distribution as

$$
\hat{\tilde{M}}_{n}(t)=n^{-1 / 2} \sum_{i=1}^{n} \sigma_{i} r\left(x_{i}, \theta_{0}\right) \exp \left(\sum_{j=1}^{d} t \xi\left(x_{i-j}\right)\right)
$$

pointwise in $t$, where $\sigma_{i}$ are bounded i.i.d. random variables independent of $x_{i}$ and $r\left(x_{i}, \theta_{0}\right)$ with $\mathrm{E}\left[\sigma_{i}^{2}\right]=1$. Thus the distribution of $\hat{M}_{n}(t)$ can be approximated based on the simulation of $n$-tuples of $\sigma_{i}$. de Jong proves that the critical regions obtained by the simulation of $\hat{\tilde{M}}_{n}(t)$ are asymptotically valid (see de Jong (1996), Theorem 5). Nevertheless, a further difficulty is that a consistent ICM test rests on the statistic $\sup _{t \in \Xi}\left|\hat{M}_{n}(t)\right|$. The calculation of this test statistic is quite cumbersome since it requires the maximization over a parameter space of dimension $n-1$. For this reason de Jong suggests to find another continuous functional of $\hat{M}_{n}(t)$ that possesses the same consistency property but is more easily calculated. Then, de Jong proposes to use the functional

$$
\Lambda=n^{-1} \int_{\Xi} \hat{M}_{n}(t)^{2} \varphi_{1}\left(t_{1}\right) d \varphi_{1} \ldots \varphi_{j}\left(t_{j}\right) d t_{j} \ldots
$$

where the integrations run over an infinite number of $t_{j}$. According to (22), each $t_{j}$ is integrated over the subset of $\mathbb{R}$, such that $a_{j} \leq\left|t_{j}\right| \leq b_{j} . \varphi_{j}(t)$ denote a sequence of density functions that integrate to one over the particular subsets. de Jong shows that the use of this functional leads to a consistent test. Since $\hat{M}_{n}(t)$ can be written as a double summation and the integrals can be calculated one at a time, we obtain a functional which is much easier to calculate than $\sup _{t \in \Xi}\left|\hat{M}_{n}(t)\right|$. By choosing a uniform distribution, i.e. $\varphi_{j}(t)=t^{-1}$, the ICM test statistic $\Lambda$ results in

$$
\begin{array}{rl}
\Lambda=n^{-1} \sum_{i=1}^{n} \sum_{j=1}^{n} & r\left(x_{i}, \theta_{0}\right) r\left(x_{j}, \theta_{0}\right) \prod_{s=1}^{d}\left\{\frac{1}{b_{j}-a_{j}}\left[\xi\left(x_{i-s}\right)+\xi\left(x_{j-s}\right)\right]^{-1}\right. \\
& \left.\times\left[\exp \left(b_{j}\left(\xi\left(x_{i-s}\right)+\xi\left(x_{j-s}\right)\right)\right)-\exp \left(a_{j}\left(\xi\left(x_{i-s}\right)+\xi\left(x_{j-s}\right)\right)\right)\right]\right\} .
\end{array}
$$

Summarizing, the implementation of ICM tests to ACD models requires the following steps:

(i) (Q)ML estimation of the ACD model: Estimate the particular ACD model by (Q)ML and calculate the conditional moment restriction $r\left(x_{i}, \theta_{0}\right)$.

(ii) Choice of $a_{j}$ and $b_{j}$ : Choose values for $a_{j}$ and $b_{j}$, defining the parameter space $\Xi$. de Jong (1996) suggests to use $a_{j}=A j^{-2}$ and $b_{j}=B j^{-2}$ where the values $A$ and $B$ $(0<A<B)$ can be chosen arbitrarily. Asymptotically the choice of $A$ and $B$ should have no influence on the power of the test, however in finite samples it probably has. Monte Carlo simulations of de Jong (1996) suggest to choose a small range, for example $A=0$ and $B=0.5$.

(iii) Choice of $\xi(\cdot)$ : According to the lemma above, the function $\xi(\cdot)$ must be a bounded one-to-one mapping from $\mathbb{R}$ into $\mathbb{R}$. Asymptotically, the choice of the function $\xi(\cdot)$ is irrelevant, however, Bierens (1990) proposes to use $\xi(x)=\arctan (x)$. In this paper we use $\xi(x)=\arctan (0.01 \cdot x) \cdot 100$ which is also a bounded function but has the 
advantage that it is nearly linear in the relevant region which improves the small sample properties of the test.

(iv) Choice of $d$ : Note that in the case of dependent data the test consistency is only ensured by accounting for all feasible lags, $d=i-1$, i.e. the dimension of the parameter space under consideration grows with the sample size. An alternative which does not require as much computer time, would be to choose a fixed value $d<n$. However, in this case the test does not allow us to consistently test the moment condition for an infinite number of conditioning variables.

(v) Simulation of $n$-tuples of $\sigma_{i}$ : Simulate $M n$-tuples of (bounded) i.i.d. random variables $\sigma_{i, m}, i=1, \ldots, n$ with $\mathrm{E}\left[\sigma_{i, m}^{2}\right]=1$ for $m=1, \ldots, M$. Following de Jong (1996), we generate the $\sigma_{i}$ variables such that $\mathrm{E}\left[\sigma_{i}=1\right]=\mathrm{E}\left[\sigma_{i}=-1\right]=0.5$.

(vi) Computation of the test statistic and simulating of the critical values:

- Compute the test statistic $\Lambda$ according to (25).

- For each $n$-tuple of $\sigma_{i}$ compute the simulated test statistic

$$
\begin{aligned}
\tilde{\Lambda}_{m}=n^{-1} \sum_{i=1}^{n} \sum_{j=1}^{n} & \left(\sigma_{i, m} r\left(x_{i}, \theta_{0}\right)\right)\left(\sigma_{j, m} r\left(x_{j}, \theta_{0}\right)\right) \prod_{s=1}^{d}\left\{\frac{1}{b_{j}-a_{j}}\left[\xi\left(x_{i-s}\right)+\xi\left(x_{j-s}\right)\right]^{-1}\right. \\
& \left.\times\left[\exp \left(b_{j}\left(\xi\left(x_{i-s}\right)+\xi\left(x_{j-s}\right)\right)\right)-\exp \left(a_{j}\left(\xi\left(x_{i-s}\right)+\xi\left(x_{j-s}\right)\right)\right)\right]\right\}, m=1, \ldots, M .
\end{aligned}
$$

(vii) Computation of simulated p-values: Since the critical region of the test has the form $(C, \infty]$, we compute the simulated p-value of the ICM test as

$$
p v_{I C M}=\frac{1}{M} \sum_{m=1}^{M} \mathbf{I}_{\left\{\tilde{\Lambda}_{m} \leq \Lambda\right\}} .
$$

\section{Monte Carlo studies}

In order to gain deeper insights into the size and power properties of the proposed diagnostic tests, we conduct an extensive Monte Carlo study. We draw samples of size 3000 which is a still relatively small sample size for high-frequency financial data. Each Monte Carlo experiment is repeated 500 times. We use 7 data generating processes (DGP's) based on different parameterizations of the ACD, LACD, BACD, EXACD, AGACD, SPACD and TACD model. We choose the persistence parameter $\beta$ to be 0.8 whereas the innovation parameters are associated with different (non-linear) news impact functions. Table 1 shows the parameterizations of the individual DGP's. Figure 1 illustrates the news response functions underlying the particular DGP's graphically. We consider convex and concave as well as clearly non-monotonous news impact curves as e.g. revealed by the SPACD and AGACD specifications. Furthermore, the DGP based on the AMACD model allows to quantify the power of the tests to detect misspecifications due to shifts of the (linear) news impact function. Finally, the DGP associated with the TACD model is chosen 
in accordance with the empirical results by Zhang, Russell, and Tsay (2001). For each replication both a (linear) $\operatorname{ACD}(1,1)$ and $\operatorname{LACD}(1,1)$ model are estimated.

Besides different types of LM, CM and ICM tests we also apply a simple alternative test. As proposed by Engle and Russell (1998), a valuable way to detect nonlinear dependencies between the ACD residuals and the past information set is to divide the residuals into bins which range from 0 to $\infty$ and to regress $\left\{e_{i}\right\}$ on indicators for the magnitude of the previous duration. A standard $F$-test on joint significance of the regressors indicates whether there is remaining prediction power implied by the bin indicators. Table 2 shows the specifications of the different tests used in this study. The LM tests are based on different forms of sign-bias variables with respect to the first two lags. For the CM tests we use two different types of conditional moment restrictions, eq. (19) and (20), and various weighting functions based on functions of past durations and innovations. Moreover, we evaluate different specifications of ICM tests. Since for the case $d=i-1$ and large samples, the calculation of the ICM test statistic and the simulated critical values is computational quite intensive, we restrict our analysis to the cases $d \in\{1,2,5,10\}$. In accordance with the simulation results by de Jong (1996), the parameters $A$ and $B$ are set to 0 and 0.5 , respectively. Finally, the ICM test statistics are computed based on $M=100$ replications. Table 3 shows the rejection rates of the particular tests based on estimations of the basic ACD $(1,1)$ model. Correspondingly, Table 4 displays the results based on estimations of the $\operatorname{LACD}(1,1)$ model. The first three parameters correspond to the median values of the $(\mathrm{L}) \mathrm{ACD}(1,1)$ parameters estimated for each replication. In both tables, the first Monte-Carlo experiment is devoted to a check of the size of the tests since the estimated model is in line with the assumed data generating process. Actually, we find evidence for significant size distortions. It turns out that the LM tests and ICM tests are clearly undersized whereas the CM and NL tests tend to be oversized in most cases. We attribute this finding to finite sample properties which seem to be still present based on a sample size of 3,000. The remaining experiments allow us to gain insights into the power properties of the particular tests. As revealed by Table 3 we find a remarkably high power by testing a basic ACD model against the EXACD, SPACD, LACD and AGACD model. Slightly lower but still high rejection rates are shown based on the AMACD model as data generating process. This result indicates that the distinction between additive and multiplicative stochastic components can be an important source of model misspecification. In contrast, violations of the conditional mean restriction implied by TACD dynamics are more difficult to detect. A similar picture is revealed by Table 4. Nevertheless, the test's power against an underlying linear ACD specification when a LACD model is estimated is clearly lower than in the reversed case. Moreover, not surprisingly, the tests have lower power to distinguish between a LACD model and an alternative specification implying also a concave news impact function. Overall, we find the highest power for conditional moment tests based 
on weighting functions which are particularly sensitive against nonlinearities in the news response function. This is still true even when we take into account that the tests tend to be oversized. Furthermore, even though the LM test has optimal power against local alternatives, it underperforms the CM test in nearly all cases. Thus for more general forms of misspecification the optimality of the LM test diminishes. Not surprisingly, the F-tests reveal a high power particularly in cases when the true data generating process implies strong nonlinearities in the news impact curve. In contrast, the test has only low power to detect violations due to neglected additive stochastic components (AMACD specification). The worst performance is shown for the ICM test. Even though this test is asymptotically consistent, its power is quite low in most cases. Only in cases of very fundamental misspecifications, we find a sufficient power. Nevertheless, in all cases the test is outperformed by the CM test.

Summarizing the Monte Carlo results, we can conclude that the CM test seems to be the most appropriate diagnostic tool to detect functional misspecification of general form even when size distortions in finite samples are taken into account. It is evident that the results are widely robust against the particular choice of the weighting function. LM tests against general forms of nonlinear news impacts underperform CM tests. However, as illustrated by Meitz and Teräsvirta (2006), the latter are quite powerful in case we want to test against particular parametric alternatives. Overall, it turns out that it is reasonable to combine these particular types of diagnostic tests to detect different forms of model misspecification.

\section{Application to NYSE financial DURATions}

In this section we apply the proposed ACD models and diagnostic tests to financial duration data from the New York Stock Exchange (NYSE). The sample covers the period from 01/02/01 to 05/30/01 and is extracted from the Trades and Quotes (TAQ) database available from the NYSE. We analyze the four intensively traded stocks AOL, Coca-Cola, Disney and GE and focus on trade durations as well as price durations. The price durations are generated as the time until a cumulated absolute price change of a given size is realized. In order to obtain price durations which are on average of comparable length we use $\$ 0.100$ midquote changes for the AOL stock and $\$ 0.050$ midquote changes for the other three stocks. In order to obtain comparable sample sizes, the trade durations are constructed using ten trading days from $03 / 19 / 01$ to $03 / 30 / 01$. Overnight spells as well as observations before 9:30 and after 16:00 are removed. In order to account for seasonality effects, which are well documented for financial durations (see for example Engle and Russell, 1998, Giot, 2000, or Gerhard and Hautsch, 2002), we use seasonally adjusted durations $\tilde{x}_{i}=x_{i} / s_{i}$, where $s_{i}$ correspond to diurnal factors which are estimated based on a regression of the plain durations on cubic splines with nodes at each 30 minutes. 
Descriptive statistics of plain durations as well as seasonally adjusted durations for the four stocks are given in Table 5 .

For each type of financial duration we estimate the ACD, LACD, BACD, EXACD, AGACD, SPACD and TACD model using QML. The lag order is chosen according to the Bayes Information Criterion (BIC). For all duration series, except for Disney and GE price durations, we find an $\operatorname{ACD}(2,1)$ parameterization to be the best specification. For the Disney and GE price durations we choose an $\operatorname{ACD}(2,2)$ specification. Note that for the nonlinear ACD specifications the inclusion of a second lag is not straightforward since it requires to parameterize also the news impact function of the latter. This leads to a doubling of the corresponding news response parameters which is not practicable especially for the highly parameterized AGACD and SPACD model. For this reason we choose a parameterization which allows us on the one hand to account for higher order dynamics but ensures on the other hand model parsimony. In particular, we model the news impact of the second lag also on the basis of the parameters $\delta_{1}, \delta_{2}$ and $b$ and double only the autoregressive parameters $\alpha, \beta, c$ and $\nu$. Then, the $\operatorname{AGACD}(2,2)$ is given by

$$
\begin{aligned}
\Psi_{i}^{\delta_{1}}=\omega & +\sum_{j=1}^{2}\left\{\alpha_{j} \Psi_{i-j}^{\delta_{1}}\left(\left|\varepsilon_{i-1}-b\right|+c_{j}\left(\varepsilon_{i-1}-b\right)\right)^{\delta_{2}}\right. \\
& \left.+\nu_{j}\left(\left|\varepsilon_{i-1}-b\right|+c_{j}\left(\varepsilon_{i-1}-b\right)\right)^{\delta_{2}}\right\}+\sum_{j=1}^{2} \beta_{j} \Psi_{i-j}^{\delta_{1}} .
\end{aligned}
$$

Applying the same principle to the SPACD model, the $\operatorname{SPACD}(2,2)$ specification is given by

$$
\begin{aligned}
\Psi_{i}=\omega & +\sum_{j=0}^{m^{+}} \alpha_{j}^{+} \mathbf{1}_{\left\{\varepsilon_{i-1}>\bar{\varepsilon}_{j}\right\}}\left(\varepsilon_{i-1}-\bar{\varepsilon}_{j}\right)+\sum_{j=0}^{m^{-}} \alpha_{j}^{-} \mathbf{1}_{\left\{\varepsilon_{i-1}<\bar{\varepsilon}_{j}\right\}}\left(\varepsilon_{i-1}-\bar{\varepsilon}_{j}\right) \\
& +\sum_{j=0}^{m^{+}}\left(\alpha_{j}^{+}+a^{+}\right) \mathbf{1}_{\left\{\varepsilon_{i-1}>\bar{\varepsilon}_{j}\right\}}\left(\varepsilon_{i-1}-\bar{\varepsilon}_{j}\right)+\sum_{j=0}^{m^{-}}\left(\alpha_{j}^{-}+a^{-}\right) \mathbf{1}_{\left\{\varepsilon_{i-1}<\bar{\varepsilon}_{j}\right\}}\left(\varepsilon_{i-1}-\bar{\varepsilon}_{j}\right)+\beta \Psi_{i-1} .
\end{aligned}
$$

Furthermore, in order to restrict the computational burden, we estimate the TACD model based on three regimes and exogenously fixed threshold values. They are fixed to the values 0.25 and 1.50 which is in accordance with the Monte Carlo study and the empirical results provided by Zhang, Russell, and Tsay (2001).

Two difficulties have to be considered: First, for nonlinear models involving absolute value functions in the news impact function the estimation of the Hessian matrix is quite cumbersome due to numerical difficulties. For this reason we estimate the asymptotic standard errors by the OPG estimator of the information matrix. Second, even though the news impact function implied by the AGACD specification allows for more flexibility, it has one major drawback since the parameter restriction $|c|<=1$ has to be imposed whenever $\delta_{2} \neq 1$ in order to circumvent complex values. Note that this restriction is binding in the case where the model implies an upward kinked concave news impact function which is 
quite typical for financial durations and which is only possible for $c<-1$ (and $\alpha<0$ ). In this case, a restricted version of the model has to be estimated. In particular, whenever $c$ converges to the boundary, either $\delta_{2}$ or, alternatively, $|c|$ has to be fixed to 1 . Setting $\delta_{2}=1$ implies a piecewise linear news impact function which is kinked at $b$. Alternatively, by setting $c=1$, the $\operatorname{AGACD}(1,1)$ specification becomes

$$
\Psi_{i}^{\delta_{1}}=\omega+\tilde{\alpha} \Psi_{i-1}^{\delta_{1}}\left(\varepsilon_{i-1}-b\right)^{\delta_{2}} \mathbf{1}_{\left\{\varepsilon_{i-1} \geq b\right\}}+\tilde{\nu}\left(\varepsilon_{i-1}-b\right)^{\delta_{2}} \mathbf{1}_{\left\{\varepsilon_{i-1} \geq b\right\}}+\beta \Psi_{i-1}^{\delta_{1}},
$$

where $\tilde{\alpha}=2^{\delta_{2}} \alpha$ and $\tilde{\nu}=2^{\delta_{2}} \nu$. Thus the news impact function is zero for $\varepsilon_{i-1} \leq b$ and follows a concave (convex) function for $\delta_{2}<1\left(\delta_{2}>1\right)$. Correspondingly, setting $c=-1$ leads to

$$
\Psi_{i}^{\delta_{1}}=\omega+\tilde{\alpha} \Psi_{i-1}^{\delta_{1}}\left(b-\varepsilon_{i-1}\right)^{\delta_{2}} \mathbf{1}_{\left\{\varepsilon_{i-1} \leq b\right\}}+\tilde{\nu}\left(b-\varepsilon_{i-1}\right)^{\delta_{2}} \mathbf{1}_{\left\{\varepsilon_{i-1} \leq b\right\}}+\beta \Psi_{i-1}^{\delta_{1}} .
$$

Note that the latter two restricted AGACD specifications do not nest the EXACD model but the BACD model for $\nu=0$ and $b=0(b \rightarrow \infty)$ whenever $c$ is set to $1(-1)$.

Tables 6, 8, 10 and 12 give the estimation results for the different types of financial durations. The following findings can be summarized: First, for trade durations the autoregressive parameters reveal only weak innovation components while the persistence parameters are close to one. In several cases even negative values for $\alpha_{1}$ are obtained, however, in these cases they are overcompensated by positive values for $\alpha_{2} \cdot{ }^{9}$ For price durations also a strong persistence is revealed while the innovation component is clearly higher than that for trade durations. ${ }^{10}$ Second, comparing the goodness-of-fit of the individual specifications based on the BIC we find the best performance for EXACD and BACD models. Especially for price durations the more simple (linear and logarithmic) models are rejected in favor of the BACD, AGACD and EXACD model. For trade durations no clear picture is revealed. While for the AOL and the Coca-Cola stock the AGACD and EXACD model is the best specification, for the Disney and the GE stock the basic ACD model leads to the highest BIC. The estimates of the TACD model provide evidence for regime-switching behavior of the autoregressive parameter, nevertheless, this specification is outperformed by the other models in terms of explanatory power. For all specifications we observe the strongest increase of the log-likelihood function when the (L)ACD model is extended to a BACD or EXACD model. This result illustrates that for both types of financial durations it is crucial to account for nonlinear news impact effects.

Third, the estimated Box-Cox parameters $\hat{\delta}_{1}$ and $\hat{\delta}_{2}$ are mostly lower than one for price durations while for trade durations both values lower and larger than one are obtained. These results lend support against the linear ACD model since we notice that price duration processes imply concave news impact curves, i.e. the adjustments of the conditional

\footnotetext{
${ }^{9}$ In this context it has to be noted that in a nonlinear ACD specification an upward kinked concave news impact function actually implies a negative value for $\alpha$, see also Section 2 .

${ }^{10}$ This finding is not surprising since price durations are aggregates of trade durations. It can be analytically shown that the innovation component increases with the aggregation level.
} 
expected mean are stronger in periods of smaller than expected price durations (volatility shocks) than in periods with low price intensities. Corresponding results are found based on the EXACD model since the mostly highly significant negative parameters $c$ imply upward kinked concave shaped news response curves. For trade durations the picture is less clear since we obtain evidence for concave as well as convex news impact curves.

Fourth, in most cases only restricted versions of the augmented ACD models are estimated since either $\delta_{1}$ tends to zero and/or $|c|$ tends to one. Since the most duration series seem to imply a concave news impact function, it is not surprising that especially the second restriction is binding for nearly all series. In the first case, the model is estimated under the restriction $\delta \rightarrow 0$ which is practically performed by estimating the model based on a logarithmic transformation. In this case, the AGACD consists only of an additive stochastic component. In the second case, two restricted versions of the model are reestimated: one specification under the restriction $|c|=1$ and one model under $\delta_{2}=1$. Then we choose the specification leading to the higher log-likelihood value. In general, we observe that the extension from the BACD/EXACD model to the AGACD model is supported by the data since we find an increase of the log-likelihood function and significant coefficients in most cases. Hence, a further flexibilization of the news impact function seems to be an important issue. Nevertheless, in some cases we also find evidence against an additive stochastic component since the parameter $\nu$ converges to zero. In this case, we fix the parameter at zero and re-estimate a restricted version of the model. In contrast, for AOL and GE trade durations we observe significant values of $\nu$ lending support for the need for additive as well as multiplicative stochastic factors. Especially for the AOL stock this extra flexibility leads to a strong increase of the log likelihood function and also the BIC value.

Fifth, the SPACD model is estimated using the categorization $\{0.1,0.25,0.5,1.0,1.5,2.0,3.0\}$ with $\bar{\varepsilon}_{0}=1.0$ which allows for high flexibility concerning very small and very large innovations. The mostly significant parameters $a^{+}$and $a^{-}$indicate that it is useful to account also for flexible news impact effects of the second lag. Figures 2 and 3 depict the news impact functions for the particular duration series computed based on the estimates of the SPACD models. The shape of the obtained news impact curves is strongly consistent with the estimates of the parametric ACD models. The news response curves for trade durations reveal high nonlinearities especially for very small innovations. For two of the stocks (AOL and Coca-Cola) we even observe a news response function that implies a downward shape for low values of $\varepsilon_{i-1}$. Hence, it turns out that for extremely small innovations (with exception of the GE stock) the first order autocorrelation is rather negative than positive. However, only for larger values of $\varepsilon_{i-1}$ the ACF is slightly positive. This finding illustrates that small durations induce significantly different adjustments of the expected mean than long durations which has be to taken into account in the econometric modelling. This 
result is in line with the findings of Zhang, Russell, and Tsay (2001) who provide evidence for similar effects based on estimations of a Threshold ACD model. Therefore, since such a news impact curve is not in accordance with symmetric ACD models, it is not surprising that the basic linear ACD model, especially for the AOL stock, is misspecified and not sufficient to model trade durations. The news response function for price durations reveals a significantly different shape. The larger (positive) slope of the curve indicates a higher (positive) autocorrelation for price durations. Nonetheless, we notice a non-linear news impact curve with a strongly increasing pattern for $\varepsilon_{i-1}<1$ and a nearly flat function for $\varepsilon_{i-1}>1$. Hence, also for price durations we observe different adjustment processes for unexpected small durations, i.e. in periods of unexpected high volatility.

Sixth, for all processes except the ACD, AGACD and TACD model, stationarity is ensured by $\sum_{j=1}^{2} \beta_{j}<1$. Correspondingly, in the ACD case the inequality $\sum_{j=1}^{2} \alpha_{j}+\beta_{j}<1$ has to be satisfied while the AGACD model implies

$$
\sum_{j=1}^{2} \beta_{j}+\alpha_{j}\left(\left|\varepsilon_{i}-b\right|+c\left(\varepsilon_{i}-b\right)\right)^{\delta_{2}}<1 .
$$

Checking these restrictions using the corresponding parameter estimates, we notice that stationarity is ensured for all considered specifications. ${ }^{11}$ The analytical derivation of the stationarity conditions for higher order TACD models is difficult and is beyond the scope of this study. ${ }^{12}$ However, own simulation studies show evidence for the stationarity of the estimated TACD processes.

The Tables 7, 9, 11 and 13 display the p-values of the applied LM, CM and ICM tests. For the LM and CM tests as well as the F-tests against nonlinearities in the news impact function we apply the same specifications as in the Monte Carlo experiments. Since for large samples the computation of the ICM test statistic is quite time consuming we use only the ICM test specifications $I C M_{1}, I C M_{2}$ and $I C M_{3}$. Note that such a test is not consistent against misspecifications with respect to a higher number of lags.

For AOL trade durations we notice that the more simple specifications are rejected on the basis of nearly all diagnostics. Especially the ACD, LACD and TACD model seem to be clearly misspecified. The more flexible models, like the BACD and EXACD specification lead to slightly higher p-values, nonetheless especially based on the CM tests the null hypothesis of a correct specification of the conditional mean is rejected for all models. Better results are obtained for the AGACD and the SPACD model. These findings are in line with the regression results that also indicate the best goodness-of-fit for these two specifications. The CM tests employing the conditional mean restriction (19) indicate a satisfying goodness-of-fit which is also supported by the corresponding $F$-tests. In contrast, CM tests using the restriction (20) still reject in most cases. However, overall, the

\footnotetext{
${ }^{11}$ Since it is cumbersome to verify the stationarity condition for the AGACD model analytically, it is evaluated based on a simulation procedure.

${ }^{12}$ Zhang, Russell, and Tsay (2001) derive the stationarity properties for the $\operatorname{TACD}(1,1)$ case.
} 
SPACD and AGACD model seem to be the most appropriate specifications, nevertheless, they still reveal deficiencies to fully capture nonlinearities in the news response. For AOL price durations we observe a clear misspecification of the ACD and LACD model since the null is rejected based on nearly all test statistics. Significantly better results are found for the BACD, EXACD and AGACD model that allow for a more flexible modelling of the (nonlinear) news impact function. Even though we find evidence for model rejections based on individual CM tests, the overall test results clearly support the more flexible ACD specifications. However, for both trade durations and price durations, we notice that the ICM test statistics indicate no rejection of the null hypothesis. Hence, the ICM test does not seem to have enough power to detect these particular forms of model misspecification. Similar results are found for Coca-Cola trade durations. Again we observe a clear rejection of the null hypothesis for the more simple models, like the ACD and LACD model. Interestingly, even the BACD model is clearly rejected. The best performance is found for the SPACD specification which seems to capture the properties in the data quite well. In contrast, based on Coca-Cola price durations effectively all specifications are rejected based on the individual CM tests. Nevertheless, the best fits are observed for the EXACD, AGACD and SPACD model. Again, the ICM test seems to have not enough power to detect model misspcecifications. Nonetheless, for the most flexible ACD parameterizations lower p-values are displayed.

A quite different picture is obtained for Disney trade durations. Here, for all models, the validity of the conditional mean restriction is not rejected. Thus the news impact curve of this duration series seem to reveal a largely linear pattern which is in accordance with more simple ACD models. This result is confirmed by the plot of the estimated news response curve (Figure 2) that actually depicts an almost linear pattern, with exception of extremely small innovations. Nonetheless, a somewhat puzzling finding is that the ICM test statistic displays misspecifications for all estimated specifications. Hence, a possible explanation could be that the LM and CM tests do not have enough power to detect the sharp drop in the upper tail of the news impact curve. For Disney price durations we find similar results as for AOL and Coca-Cola price durations. We notice again a clear rejection of the ACD and LACD model while the higher parameterized models induce higher p-values of the individual tests. Here, the AGACD model is the horse race winner. Nevertheless, as indicated by the low p-values of the corresponding CM and LM tests, the augmented ACD specifications still seem not to be able to fully capture the existing asymmetric news impact effects in the data.

The diagnostic results for GE financial durations are similar to the findings for the AOL and Coca-Cola stock. While the more simple models are largely rejected, it turns out that the more sophisticated specifications of the conditional mean function yield additional reward as they seem to be more appropriate to pass the individual tests. The overall best 
diagnostic results are again achieved by the AGACD and NPACD model. Nonetheless, the $\mathrm{CM}$ test statistics still indicate rejections of the null hypothesis of correct model misspecification due to unconsidered asymmetries in the news response.

Summarizing the diagnostic results based on the investigated financial duration series we conclude the following findings: First, overall it seems to be easier to model trade durations than price durations. This finding is also supported by the graphical illustrations of the estimated news impact functions. It clearly turns out that asymmetric news impact effects are much more apparent for price durations than for trade durations. Second, the overall best performance in terms of a valid conditional mean restriction is obtained by the AGACD and SPACD model which allow for quite flexible parameterizations of the news impact curve. Third, it turns out that nonlinear news response effects exist not only for the first lag but also for higher order lags which has to be taken into account in the econometric specification. Fourth, LM tests are more conservative than CM tests. These findings confirm the results of the Monte-Carlo study. Overall, the CM tests clearly indicate the highest power to detect potential misspecificatios of the underlying model. Slight evidence is found that the conditional moment restriction (20) induces a higher power. However, as indicated by the Monte-Carlo studies, some of the CM tests are oversized which should be taken into account when the sample size is small. Nevertheless, our results clearly show the usefulness of these tests to detect model misspecifications. Fifth, in nearly all cases ICM tests seem to have not enough power. Given the computational effort which is needed to compute the ICM test statistics (particularly for long time series), their benefit is questionable compared to traditional CM tests.

\section{Conclusions}

This paper suggests and analyzes different types of specification tests to test the validity of the conditional mean restriction implied by ACD models. Three types of tests are suggested: Lagrange Multiplier (LM) tests against sign bias alternatives, conditional moment $(\mathrm{CM})$ tests based on various types of weighting functions, and integrated conditional moment (ICM) tests based on an infinite number of conditional moment restrictions as omnibus tests against arbitrary forms of model misspecification. The proposed testing framework is applied to a wide range of existing and new ACD specifications including augmented ACD models based on additive and multiplicative stochastic components, threshold ACD models, and a new type of ACD model based on a semiparametric specification of the news response function. In order to examine the power of the particular tests we conduct an extensive Monte-Carlo experiment. We find evidence for size distortions in finite samples. In particular, LM tests seem to be undersized whereas CM tests tend to be oversized. The highest power is provided by $\mathrm{CM}$ tests based on weighting functions which include sign bias variables and are sensitive against nonlinearities in the news response 
function. Despite of their consistency, ICM tests have the lowest power and seem to be undersized in finite samples.

The individual ACD models and tests are applied to trade durations and price durations of the AOL, Coca-Cola, Disney and GE stock traded at the NYSE. We find evidence for strong nonlinearities in the news impact function leading to clear rejections of linear and logarithmic ACD specifications. In particular, trade durations reveal a convex news response curve which is downward shaped for small innovations and follows an upward shape for high innovations. For price durations a concave news impact function is observed which is strongly increasing for small durations and nearly constant for durations larger than expected. While more simple ACD specifications imply clear violations of the conditional mean restriction, the overall best performance is obtained by flexible augmented ACD models and semiparametric ACD models. In general, trade durations seem to be easier modelled than price durations. Especially for price durations, even highly parameterized ACD models are not flexible enough to capture nonlinearities in the news response and to ensure a valid conditional mean specification.

Three major conclusions can be drawn based on this study: First, it turns out there is actually need to test the validity of the functional form of ACD models since it is shown that especially more simple models imply clear violations of the conditional mean restriction. Second, CM tests seem to be a very useful diagnostic tool to detect misspecifications of the model. In contrast, the additional benefit of ICM tests is limited given the low power in small samples and the high computational effort required to compute these tests. Third, there is necessity for ACD models which allow for nonlinear news response functions. The empirical results point out that in particular very small innovations associated with unexpected high active trading periods imply different updates of the conditionally expected mean. Our results show that a semiparametric specification of the news impact function as implied by the semiparametric ACD model proposed in this paper provides a valuable starting point in order to appropriately capture the statistical properties of financial duration processes.

\section{REFERENCES}

Admati, A., AND P. Pfleiderer (1988): "A Theory of Intraday Patterns: Volume and Price Variability," Review of Financial Studies, 1, 3-40.

Baumens, L., P. Giot, J. Grammig, And D. Veredas (2004): "A Comparison of Financial Duration Models Via Density Forecasts," International Journal of Forecasting, 20, 589-609.

Bauwens, L., And P. Giot (2000): "The Logarithmic ACD Model: An Application to the Bid/Ask Quote Process of two NYSE Stocks," Annales d'Economie et de Statistique, 60, 117-149.

Bierens, H. J. (1982): "Consistent Model Specification Tests," Journal of Econometrics, 20, 105-134. 1458 .

Bollerslev, T., And J. Wooldridge (1992): "Quasi-Maximum Likelihood Estimation and Inference in Dynamic Models with Time Varying Covariances," Econometric Reviews, 11, 143-172.

Breusch, T. S., AND A. R. PAgan (1979): "A Simple Test for Heteroskedasticity and Random Coefficient Variation," Econometrica, 47, 203-207. 
Breusch, T. S. (1978): "Testing for Autocorrelation in Dynamic Linear Models," Australian Economic Papers, 17, 334-355.

Carrasco, M., And X. Chen (2002): "Mixing and Moment Properties of various GARCH and Stochastic Volatility Models," Econometric Theory, 18(1), 17-39.

DE Jong, R. M. (1996): "The Bierens Test under Data Dependence," Journal of Econometrics, 72, 1-32. Diamond, D. W., And R. E. Verrecchia (1987): "Constraints on Short-Selling and Asset Price Adjustment to Private Information," Journal of Financial Economics, 18, 277-311.

Dufour, A., AND R. F. Engle (2000): "The ACD Model: Predictability of the Time between Consecutive Trades," Working Paper, ISMA Centre, University of Reading.

Easley, D., AND M. O‘Hara (1992): "Time and Process of Security Price Adjustment," The Journal of Finance, 47, 577-605.

Engle, R. F., AND V. K. NG (1993): "Measuring and Testing the Impact of News on Volatility," Journal of Finance, 48, 1749-1778.

Engle, R. F., AND J. R. Russell (1994): "Forecasting Transaction Rates: The Autoregressive Conditional Duration Model," Discussion Paper 4966, University of California San Diego.

- (1998): "Autoregressive Conditional Duration: A New Model for Irregularly Spaced Transaction Data," Econometrica, 66, 1127-1162.

Engle, R. F. (1984): "Wald, Likelihood Ratio and Lagrange Multiplier Tests in Econometrics," in Handbook of Econometrics, Vol. II, ed. by Z. Griliches, and M. D. Intriligator, chap. 13, pp. 775-826. Elsevier Science.

Engle, R. F. (2000): "The Econometrics of Ultra-High-Frequency Data," Econometrica, 68, 1, 1-22.

Fernandes, M., AND J. Grammig (2005): "Non-parametric Specification Tests for Conditional Duration Models," Journal of Econometrics, 127, 35-68.

Fernandes, M., AND J. Grammig (2006): "A Family of Autoregressive Conditional Duration Models," Journal of Econometrics, 130, 1-23.

Gerhard, F., AND N. Hautsch (2002): "Volatility Estimation on the Basis of Price Intensities," Journal of Empirical Finance, 9, 57-89.

Giot, P. (2000): "Time Transformations, Intraday Data and Volatility Models," Journal of Computational Finance, 4(2), 31-62.

Godfrey, L. G. (1978): "Testing against General Autoregressive and Moving Average Error Models when the Regressors include Lagged Dependent Variables," Econometrica, 46, 1293-1302.

Hautsch, N. (2003): "Assessing the Risk of Liquidity Suppliers on the Basis of Excess Demand Intensities," Journal of Financial Econometrics, 1, 189-215.

Hentschel, L. (1995): "All in the Family: Nesting Symmetric and Asymmetric GARCH Models," Journal of Financial Economics, 39, 71-104.

JASIAK, J. (1998): "Persistence in Intratrade Durations," Finance, 19, 166-195.

Meitz, M., AND T. TerÄsvirta (2006): "Evaluating Models of Autoregressive Conditional Duration," Journal of Business \& Economic Statistics, 24, 104-124.

Moknadem, A. (1990): "Propriétés de mélange des modèles autoregressifs polynomiaux," Annales de l'Institut Henri Poincaré, 26, 219-260.

NELSON, D. (1991): "Conditional Heteroskedasticity in Asset Returns: A New Approach," Journal of Econometrics, 43, 227-251.

Newey, W. (1985): "Maximum Likelihood Specification Testing and Conditional Moment Tests," Econometrica, 5, 1047-1070.

Rabemananjara, J., And J. M. Zakoian (1993): "Threshold ARCH Models and Asymmetries in Volatility," Journal of Applied Econometrics, 8, 31-49.

Wooldridge, J. M. (1990): "A Unified Approach to Robust, Regression-Based Specification Tests," Econometric Theory.

Zakoian, J. M. (1994): "Threshold Heteroskedastic models," Journal of Economic Dynamics and Control, $18,931-955$.

Zhang, M. Y., J. Russell, And R. S. Tsay (2001): "A Nonlinear Autoregressive Conditional Duration Model with Applications to Financial Transaction Data," Journal of Econometrics, 104, 179-207. 


\section{APPENDIX}

\section{A.1. Monte Carlo Studies.}

Table 1: Assumed data generating processes in the Monte-Carlo study. Categories for the SPACD model: $0.1,0.2, \ldots, 2.0$, where $\bar{\varepsilon}_{0}=1.0$. Threshold values for the three-regime TACD model: 0.25 and 1.50 .

\begin{tabular}{|c|c|c|c|c|c|c|c|}
\hline & $\begin{array}{c}(1) \\
\mathrm{ACD}\end{array}$ & $\begin{array}{c}(2) \\
\text { EXACD }\end{array}$ & $\begin{array}{c}(3) \\
\text { AMACD }\end{array}$ & $\begin{array}{c}(4) \\
\text { SPACD }\end{array}$ & $\begin{array}{c}(5) \\
\text { TACD }\end{array}$ & $\begin{array}{c}(6) \\
\text { LACD }\end{array}$ & $\begin{array}{c}(7) \\
\text { AGACD }\end{array}$ \\
\hline$\omega$ & 0.100 & -0.180 & 0.000 & 0.237 & & 0.137 & 0.010 \\
\hline$\alpha$ & 0.100 & 0.500 & 0.050 & -0.100 & & 0.300 & 0.100 \\
\hline$\beta$ & 0.800 & 0.800 & 0.800 & 0.800 & & 0.800 & 0.800 \\
\hline$\delta_{1}$ & & & & & & & 0.800 \\
\hline$\delta_{2}$ & & & & & & & 1.500 \\
\hline$\nu$ & & & 0.500 & & & & 0.200 \\
\hline$c$ & & & & & & & -0.300 \\
\hline$\delta_{1}$ & & & & & & & 0.500 \\
\hline$\delta_{2}$ & & & & & & & 1.500 \\
\hline$b$ & & & & & & & 0.600 \\
\hline$\alpha_{1}$ & & & & 0.300 & & & \\
\hline$\alpha_{2}^{+}$ & & & & -0.200 & & & \\
\hline$\alpha_{3}^{+}$ & & & & -0.200 & & & \\
\hline$\alpha_{4}^{+}$ & & & & -0.150 & & & \\
\hline$\alpha_{5}^{+}$ & & & & 0.000 & & & \\
\hline$\alpha_{6}^{+}$ & & & & 0.050 & & & \\
\hline$\alpha_{7}^{+}$ & & & & 0.000 & & & \\
\hline$\alpha_{8}^{+}$ & & & & 0.050 & & & \\
\hline$\alpha_{9}^{+}$ & & & & 0.100 & & & \\
\hline$\alpha_{10}^{+}$ & & & & 0.200 & & & \\
\hline$\alpha_{1}^{-}$ & & & & 0.700 & & & \\
\hline$\alpha_{2}^{-}$ & & & & 0.100 & & & \\
\hline$\alpha_{3}^{-}$ & & & & 0.100 & & & \\
\hline$\alpha_{4}^{-}$ & & & & 0.000 & & & \\
\hline$\alpha_{5}^{-}$ & & & & 0.100 & & & \\
\hline$\alpha_{6}^{-}$ & & & & 0.000 & & & \\
\hline$\alpha_{7}^{-}$ & & & & 0.500 & & & \\
\hline$\alpha_{8}^{-}$ & & & & -0.500 & & & \\
\hline$\alpha_{9}^{-}$ & & & & -2.000 & & & \\
\hline$\alpha_{10}^{-}$ & & & & -7.000 & & & \\
\hline$\omega^{1}$ & & & & & 0.050 & & \\
\hline$\alpha^{1}$ & & & & & 0.200 & & \\
\hline$\beta^{1}$ & & & & & 0.850 & & \\
\hline$\omega^{2}$ & & & & & 0.100 & & \\
\hline$\alpha^{2}$ & & & & & 0.050 & & \\
\hline$\beta^{2}$ & & & & & 0.900 & & \\
\hline$\omega^{3}$ & & & & & 0.200 & & \\
\hline$\alpha^{3}$ & & & & & 0.030 & & \\
\hline$\beta^{3}$ & & & & & 0.800 & & \\
\hline
\end{tabular}



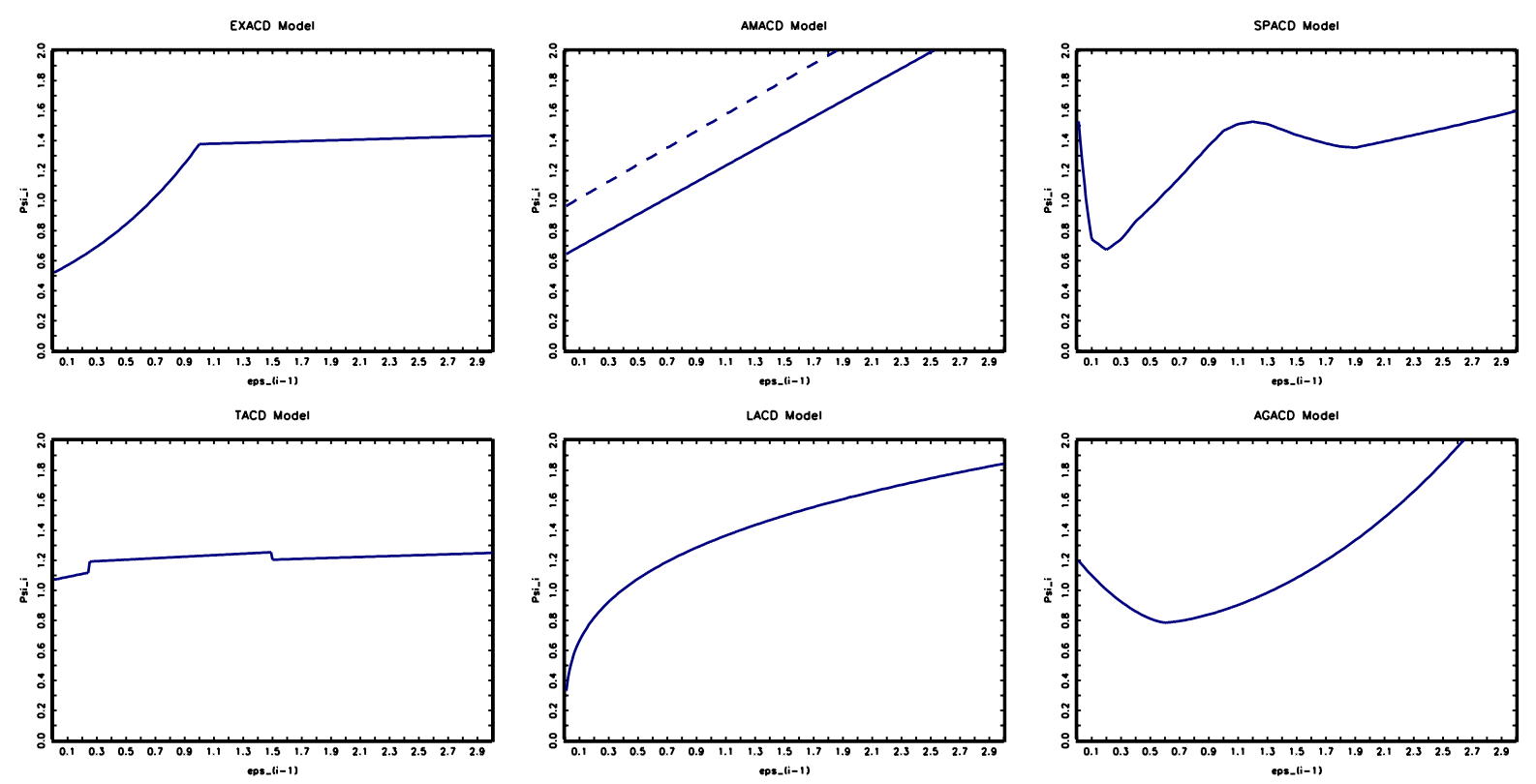

Figure 1: News impact curves underlying the different data generating processes used in the Monte-Carlo studies.

Table 2: Parameterizations of the specification tests used in the Monte-Carlo studies and in the empirical applications.

\begin{tabular}{|c|c|}
\hline \multicolumn{2}{|r|}{ LM tests } \\
\hline$L M_{1}$ & $z_{a i}^{1}=\left(\mathbb{1}_{\left\{\varepsilon_{i-1}<1\right\}}, \mathbb{1}_{\left\{\varepsilon_{i-1}<1\right\}} \varepsilon_{i-1}, \mathbb{1}_{\left\{\varepsilon_{i-1} \geq 1\right\}} \varepsilon_{i-1}\right)^{\prime}$ \\
\hline$L M_{2}$ & $z_{a i}^{2}=\left(z_{a i}^{1 \prime}, \mathbb{1}_{\left\{\varepsilon_{i-2}<1\right\}}, \mathbb{1}_{\left\{\varepsilon_{i-2}<1\right\}} \varepsilon_{i-2}, \mathbb{1}_{\left\{\varepsilon_{i-2} \geq 1\right\}} \varepsilon_{i-2},\right)^{\prime}$ \\
\hline$L M_{3}$ & $z_{a i}^{3}=\left(\mathbb{1}_{\left\{x_{i-1}<1\right\}}, \mathbb{1}_{\left\{x_{i-1}<1\right\}} x_{i-1}, \mathbb{1}_{\left\{x_{i-1} \geq 1\right\}} x_{i-1}\right)^{\prime}$ \\
\hline$L M_{4}$ & $z_{a i}^{4}=\left(z_{a i}^{3 \prime}, \mathbb{1}_{\left\{x_{i-2}<1\right\}}, \mathbb{1}_{\left\{x_{i-2}<1\right\}} x_{i-2}, \mathbb{1}_{\left\{x_{i-2} \geq 1\right\}} x_{i-2},\right)^{\prime}$ \\
\hline$L M_{5}$ & $z_{a i}^{5}=\left(z_{a i}^{1 \prime}, z_{a i}^{3 \prime},\right)^{\prime}$ \\
\hline$L M_{6}$ & $z_{a i}^{6}=\left(z_{a i}^{2 \prime}, z_{a i}^{4 \prime},\right)^{\prime}$ \\
\hline$L M_{7}$ & bins for $\varepsilon_{i-1}$ and $\varepsilon_{i-2}:[0,0.1),[0.1,0.2),[0.2,0.5),[0.5,0.8),[0.8,1),[1.2,1.5),[1.5,2),[2,3),[3, \infty)$ \\
\hline$L M_{8}$ & bins for $\varepsilon_{i-1}$ and $\varepsilon_{i-2}:[0,0.1),[0.1,0.2),[0.2,0.5),[0.5,0.8),[0.8,1),[1.2,1.5),[1.5,2),[2,3),[3, \infty)$ \\
\hline \multicolumn{2}{|r|}{ F-tests against nonlinearities in the news response } \\
\hline$N L_{1}$ & bins for $\varepsilon_{i-1}:[0,0.1),[0.1,0.2),[0.2,0.5),[0.5,0.8),[0.8,1),[1.2,1.5),[1.5,2),[2,3),[3, \infty)$ \\
\hline$N L_{2}$ & bins for $\varepsilon_{i-1}$ and $\varepsilon_{i-2}:[0,0.1),[0.1,0.2),[0.2,0.5),[0.5,0.8),[0.8,1),[1.2,1.5),[1.5,2),[2,3),[3, \infty)$ \\
\hline$N L_{3}$ & bins for $x_{i-1}:[0,0.1),[0.1,0.2),[0.2,0.5),[0.5,0.8),[0.8,1),[1.2,1.5),[1.5,2),[2,3),[3, \infty)$ \\
\hline$N L_{4}$ & bins for $x_{i-1}$ and $x_{i-2}:[0,0.1),[0.1,0.2),[0.2,0.5),[0.5,0.8),[0.8,1),[1.2,1.5),[1.5,2),[2,3),[3, \infty)$ \\
\hline \multicolumn{2}{|r|}{ CM tests } \\
\hline$C M^{1}$ & $r(\cdot)=e_{i}-1$ \\
\hline$C M^{2}$ & $r(\cdot)=x_{i}-\hat{\Psi}_{i}$ \\
\hline$C M_{1}$ & $w_{1}(\cdot)=\left(x_{i-1}, x_{i-1}^{2}, x_{i-1}^{3}, e_{i-1}, e_{i-1}^{2}, e_{i-1}^{3}\right)^{\prime}$ \\
\hline$C M_{2}$ & $w_{2}(\cdot)=\left(w_{1}(\cdot)^{\prime}, x_{i-2}, x_{i-2}^{2}, x_{i-2}^{3}, e_{i-2} e_{i-2}^{2}, e_{i-2}^{3}\right)^{\prime}$ \\
\hline$C M_{3}$ & $w_{3}(\cdot)=\left(x_{i-1}, z_{a i}^{1 \prime}\right)^{\prime}$ \\
\hline$C M_{4}$ & $w_{4}(\cdot)=\left(x_{i-1}, x_{i-2}, z_{a i}^{2 \prime}\right)^{\prime}$ \\
\hline$C M_{5}$ & $w_{5}(\cdot)=\left(e_{i-1}, z_{a i}^{3 \prime}\right)^{\prime}$ \\
\hline$C M_{6}$ & $w_{6}(\cdot)=\left(e_{i-1}, e_{i-2}, z_{a i}^{4 \prime}\right)^{\prime}$ \\
\hline$C M_{7}$ & $w_{7}(\cdot)=\left(z_{a i}^{1 \prime}, z_{a i}^{3 \prime},\right)^{\prime}$ \\
\hline$C M_{8}$ & $w_{8}(\cdot)=\left(z_{a i}^{2 \prime}, z_{a i}^{4 \prime},\right)^{\prime}$ \\
\hline$C M_{9}^{\dot{2}}$ & $w_{9}(\cdot)=\left(x_{i-1}, x_{i-2}, \ldots, x_{i-10}\right)^{\prime}$ \\
\hline$C M_{10}$ & $w_{1} 0(\cdot)=\left(e_{i-1}, e_{i-2}, \ldots, e_{i-10}\right)^{\prime}$ \\
\hline$C M_{11}$ & bins for $\varepsilon_{i-1}$ and $\varepsilon_{i-2}:[0,0.1),[0.1,0.2),[0.2,0.5),[0.5,0.8),[0.8,1),[1.2,1.5),[1.5,2),[2,3),[3, \infty)$ \\
\hline$C M_{12}$ & bins for $x_{i-1}$ and $x_{i-2}:[0,0.1),[0.1,0.2),[0.2,0.5),[0.5,0.8),[0.8,1),[1.2,1.5),[1.5,2),[2,3),[3, \infty)$ \\
\hline \multicolumn{2}{|r|}{ ICM tests } \\
\hline$I C M_{1}$ & $r(\cdot)=x_{i}-\hat{\Psi}_{i}, \quad w(\cdot)=1, \quad A=0, \quad B=0.5, \quad M=100 \quad d=1$ \\
\hline $\mathrm{ICM}_{2}$ & $r(\cdot)=x_{i}-\hat{\Psi}_{i}, \quad w(\cdot)=1, \quad A=0, \quad B=0.5, \quad M=100 \quad d=2$ \\
\hline$I C M_{3}$ & $r(\cdot)=x_{i}-\hat{\Psi}_{i}, \quad w(\cdot)=1, \quad A=0, \quad B=0.5, \quad M=100 \quad d=5$ \\
\hline $\mathrm{ICM}_{4}$ & $r(\cdot)=x_{i}-\hat{\Psi}_{i}, \quad w(\cdot)=1, \quad A=0, \quad B=0.5, \quad M=100 \quad d=10$ \\
\hline
\end{tabular}


Table 3: Rejection frequencies of the applied diagnostic tests (see Table 2) based on the data generating processes shown in Table 1. Size of simulated samples: 3000. Number of replications: 500. Estimated model: $\operatorname{ACD}(1,1)$.

\begin{tabular}{|c|c|c|c|c|c|c|c|c|c|c|c|c|c|c|}
\hline & \multicolumn{2}{|c|}{$\mathrm{ACD}$} & \multicolumn{2}{|c|}{ EXACD } & \multicolumn{2}{|c|}{ AMACD } & \multicolumn{2}{|c|}{ SPACD } & \multicolumn{2}{|c|}{ TACD } & \multicolumn{2}{|c|}{ LACD } & \multicolumn{2}{|c|}{ AGACD } \\
\hline & $5 \%$ & $10 \%$ & $5 \%$ & $10 \%$ & $5 \%$ & $10 \%$ & $5 \%$ & $10 \%$ & $5 \%$ & $10 \%$ & $5 \%$ & $10 \%$ & $5 \%$ & $10 \%$ \\
\hline \multicolumn{15}{|c|}{ Median of estimated $\operatorname{ACD}(1,1)$ parameters } \\
\hline$\omega$ & 0.107 & 0.107 & 0.159 & 0.159 & 0.466 & 0.466 & 0.174 & 0.174 & 0.188 & 0.188 & .147 & 0.147 & 0.061 & 0.061 \\
\hline$\alpha$ & .101 & .101 & 491 & 491 & 0.255 & 0.255 & 0.310 & 0.310 & .042 & 0.042 & .488 & 0.488 & .476 & 0.476 \\
\hline$\beta$ & 0.792 & 0.792 & .387 & 0.387 & 0.646 & 0.646 & 0.525 & 0.525 & 0.799 & 0.799 & 0.401 & 0.401 & .496 & 0.496 \\
\hline \multicolumn{15}{|c|}{ LM tests } \\
\hline$L M_{1}$ & 0.002 & 0.014 & 1.000 & 1.000 & 0.021 & 0.038 & 0.242 & 0.348 & 0.028 & 0.050 & .970 & 0.984 & 1.000 & 1.000 \\
\hline$L M_{2}$ & .012 & .022 & 998 & .000 & 0.058 & 0.096 & 0.460 & 0.550 & 0.044 & 0.078 & .996 & 0.998 & .000 & 1.000 \\
\hline$L M_{3}$ & 0.008 & 0.026 & .998 & 0.998 & 0.027 & 0.031 & 0.284 & 0.402 & 0.042 & 0.070 & 1.000 & 1.000 & .106 & 0.158 \\
\hline$M_{4}$ & 010 & .022 & 000 & 000 & 0.058 & 0.072 & 0.434 & 0.568 & .074 & 0.102 & 1.000 & 1.000 & .106 & 0.166 \\
\hline$L M_{5}$ & 008 & .034 & 000 & .000 & 0.069 & 0.151 & 0.316 & 0.440 & 0.028 & 0.074 & .000 & 1.000 & .998 & 1.000 \\
\hline$L M_{6}$ & 014 & 040 & 000 & .000 & 0.165 & 0.244 & 0.442 & 0.588 & 0.056 & 0.108 & .000 & 1.000 & .000 & 1.000 \\
\hline$L M_{7}$ & 0.032 & 0.052 & .986 & 0.994 & 0.031 & 0.093 & 0.996 & 0.998 & 0.086 & 0.156 & 0.992 & 0.996 & 1.000 & 1.000 \\
\hline & & 0.044 & & 0.992 & 0.048 & 0.076 & 0.902 & 0.954 & 0.084 & 0.150 & 1.000 & 1.000 & .560 & 0.700 \\
\hline \multicolumn{15}{|c|}{ CM tests } \\
\hline$C M_{1}^{1}$ & 0.066 & .126 & .000 & .000 & 0.498 & 0.605 & 0.578 & 0.680 & 0.140 & 0.212 & 1.000 & 1.000 & 0.996 & 0.996 \\
\hline$C M_{2}^{1}$ & .076 & .142 & .000 & 1.000 & 0.526 & 0.670 & 0.640 & 0.740 & 0.132 & 0.210 & 0.994 & 1.000 & 0.890 & 0.936 \\
\hline$M_{3}^{1}$ & 074 & 146 & 000 & .000 & 0.454 & 0.591 & 0.630 & 0.748 & 0.156 & 0.250 & 1.000 & 1.000 & .000 & 1.000 \\
\hline$C M_{4}^{1}$ & 0.070 & .148 & 1.000 & 1.000 & 0.443 & 0.584 & 0.638 & 0.764 & 0.162 & 0.246 & 1.000 & 1.000 & .934 & 0.956 \\
\hline$C M_{5}^{1}$ & 0.068 & 0.138 & 1.000 & .000 & 0.464 & 0.581 & 0.754 & 0.838 & 0.168 & 0.254 & 1.000 & 1.000 & 1.000 & 1.000 \\
\hline$C M_{6}^{1}$ & 0.064 & 0.136 & 1.000 & 1.000 & 0.436 & 0.584 & 0.830 & 0.894 & 0.162 & 0.266 & 1.000 & 1.000 & 0.966 & 0.972 \\
\hline$M_{7}^{1}$ & .074 & .116 & .000 & 1.000 & 0.485 & 0.591 & 0.834 & 0.896 & 0.182 & 0.282 & 1.000 & 1.000 & 1.000 & 1.000 \\
\hline$C M_{8}^{1}$ & 0.076 & 130 & .000 & 1.000 & 0.447 & 0.567 & 0.856 & 0.916 & 0.186 & 0.284 & 1.000 & 1.000 & 0.976 & 0.990 \\
\hline$C M_{9}^{1}$ & 0.072 & .120 & 1.000 & .000 & 0.488 & 0.601 & 0.634 & 0.748 & 0.168 & 0.274 & 0.998 & 1.000 & 0.998 & 1.000 \\
\hline$C M_{10}^{1}$ & 0.068 & .122 & .000 & .000 & 0.440 & 0.564 & 0.650 & 0.752 & 0.188 & 0.286 & 0.996 & 1.000 & 0.882 & 0.936 \\
\hline$C M_{11}^{1}$ & 0.064 & 0.104 & 1.000 & 1.000 & 0.519 & 0.615 & 0.716 & 0.820 & 0.210 & 0.314 & 1.000 & 1.000 & 1.000 & 1.000 \\
\hline$C M_{12}^{1}$ & 0.066 & 0.126 & 1.000 & .000 & 0.450 & 0.574 & 0.706 & 0.794 & 0.222 & 0.338 & 1.000 & 1.000 & 0.958 & 0.974 \\
\hline$C M_{1}^{2}$ & 0.070 & .116 & 1.000 & 1.000 & 0.419 & 0.529 & 0.786 & 0.860 & 0.154 & 0.256 & 1.000 & 1.000 & 1.000 & 1.000 \\
\hline$C M_{2}^{2}$ & 0.064 & .128 & .000 & .000 & 0.388 & 0.495 & 0.856 & 0.930 & 0.156 & 0.260 & 1.000 & 1.000 & 0.984 & 0.994 \\
\hline$C M_{3}^{2}$ & 0.078 & 0.154 & 1.000 & .000 & 0.440 & 0.560 & 0.828 & 0.896 & 0.174 & 0.282 & 1.000 & 1.000 & 1.000 & 1.000 \\
\hline$C M_{4}^{2}$ & 0.080 & 0.156 & 1.000 & .000 & 0.368 & 0.522 & 0.890 & 0.934 & 0.174 & 0.306 & 1.000 & 1.000 & 0.990 & 1.000 \\
\hline$C M_{5}^{2}$ & 0.068 & .120 & 0.998 & 1.000 & 0.471 & 0.588 & 0.604 & 0.708 & 0.090 & 0.150 & 0.988 & 0.996 & 0.812 & 0.884 \\
\hline$C M_{6}^{2}$ & 0.070 & .122 & 0.972 & .988 & 0.478 & 0.598 & 0.596 & 0.708 & 0.098 & 0.178 & 0.952 & 0.980 & 0.526 & 0.640 \\
\hline$C M_{7}^{2}$ & 0.068 & 0.128 & .000 & .000 & 0.117 & 0.199 & 0.406 & 0.516 & 0.074 & 0.150 & 1.000 & 1.000 & 0.990 & 0.992 \\
\hline & 0.060 & 0.122 & 000 & .000 & 0.388 & 0.519 & 0.622 & 0.722 & 0.102 & 0.170 & 0.998 & 0.998 & 0.756 & 0.846 \\
\hline$M_{9}^{2}$ & 0.108 & .168 & 000 & .000 & 0.103 & 0.141 & 1.000 & 1.000 & 0.218 & 0.348 & 1.000 & 1.000 & 1.000 & 1.000 \\
\hline$C M_{10}^{2}$ & 0.098 & .174 & 000 & .000 & 192 & 0.326 & 1.000 & 1.000 & 0.222 & 0.338 & 1.000 & 1.000 & 0.998 & 1.000 \\
\hline$C M_{11}^{2}$ & 0.094 & 0.156 & 0.976 & .988 & 0.082 & 0.117 & 0.980 & 0.992 & 0.228 & 0.350 & 1.000 & 1.000 & 1.000 & 1.000 \\
\hline$C M_{12}^{2}$ & 0.082 & 0.180 & 0.998 & 1.000 & 0.261 & 0.392 & 0.996 & 0.998 & 0.232 & 0.354 & 1.000 & 1.000 & 0.974 & 0.992 \\
\hline \multicolumn{15}{|c|}{ F-tests against nonlinearites in the news response } \\
\hline$N L_{1}$ & .064 & .136 & 000 & 1.000 & 0.052 & 0.110 & 1.000 & 1.000 & 0.166 & 0.266 & 1.000 & 1.000 & 1.000 & 1.000 \\
\hline & 0.062 & .138 & 000 & 000 & 058 & 0.113 & 1.000 & 1.000 & 0.164 & 0.258 & 1.000 & 1.000 & 1.000 & 1.000 \\
\hline & .080 & 0.154 & .928 & 0.964 & 0.065 & 0.120 & 0.972 & 0.990 & 0.170 & 0.292 & 0.998 & 0.998 & 0.818 & 0.890 \\
\hline$N L_{4}$ & & 0.126 & & & & & 0.990 & & 0.194 & 0.322 & 0.998 & 1.000 & 0.810 & 0.896 \\
\hline \multicolumn{15}{|c|}{ ICM tests } \\
\hline$I C M_{1}$ & & & & & & & & 0. & 0.014 & 0.0 & .840 & 0.872 & .202 & 0.288 \\
\hline $\mathrm{ICM}_{2}$ & 0.008 & 0.020 & & 0.940 & 0.203 & 0.275 & & 0.552 & 0.014 & 0.034 & 0.822 & 0.860 & 0.226 & 0.302 \\
\hline $\mathrm{ICM}_{3}$ & 0.010 & 0.022 & 0.908 & 0.930 & 0.199 & 0.306 & 0.492 & 0.576 & 0.012 & 0.030 & 0.824 & 0.860 & 0.244 & 0.328 \\
\hline $\mathrm{ICM}_{4}$ & 0.006 & 0.022 & 0.912 & 0.934 & 0.199 & 0.316 & 0.488 & 0.578 & 0.016 & 0.030 & 0.818 & 0.852 & 0.250 & 0.340 \\
\hline
\end{tabular}


Table 4: Rejection frequencies of the applied diagnostic tests (see Table 2) based on the data generating processes shown in Table 1. Size of simulated samples: 3000. Number of replications: 500. Estimated model: $\operatorname{LACD}(1,1)$.

\begin{tabular}{|c|c|c|c|c|c|c|c|c|c|c|c|c|c|c|}
\hline & \multicolumn{2}{|c|}{ LACD } & \multicolumn{2}{|c|}{ EXACD } & \multicolumn{2}{|c|}{ AMACD } & \multicolumn{2}{|c|}{ SPACD } & \multicolumn{2}{|c|}{ TACD } & \multicolumn{2}{|c|}{$\mathrm{ACD}$} & \multicolumn{2}{|c|}{ AGACD } \\
\hline & $5 \%$ & $10 \%$ & $5 \%$ & $10 \%$ & $5 \%$ & $10 \%$ & $5 \%$ & $10 \%$ & $5 \%$ & $10 \%$ & $5 \%$ & $10 \%$ & $5 \%$ & $10 \%$ \\
\hline \multicolumn{15}{|c|}{ Median of estimated $\operatorname{ACD}(1,1)$ parameters } \\
\hline$\omega$ & 0.101 & 0.101 & 0.120 & 0.120 & 0.254 & 0.254 & 0.078 & 0.078 & 0.043 & 0.043 & 0.036 & 0.036 & 0.154 & 0.154 \\
\hline$\alpha$ & 100 & .100 & 256 & 256 & .132 & 0.132 & 0.144 & 0.144 & 0.035 & 0.035 & .065 & 0.065 & .260 & 0.260 \\
\hline$\beta$ & 0.795 & 0.795 & .800 & 0.800 & 0.847 & 0.847 & 0.814 & 0.814 & 0.880 & 0.880 & 0.888 & 0.888 & .848 & 0.848 \\
\hline \multicolumn{15}{|c|}{ LM tests } \\
\hline$L M_{1}$ & 0.010 & 0.020 & 0.032 & 0.080 & 0.676 & 0.814 & 0.938 & 0.970 & 0.008 & 0.013 & .112 & 0.194 & 0.996 & 0.998 \\
\hline$L M_{2}$ & 012 & .026 & 064 & 120 & 891 & 0.929 & 0.978 & 0.990 & 0.020 & 0.035 & .218 & 0.362 & .000 & 1.000 \\
\hline$L M_{3}$ & 0.008 & 0.016 & .044 & 0.096 & 0.579 & 0.707 & 0.948 & 0.980 & 0.005 & 0.020 & 0.110 & 0.194 & .976 & 0.990 \\
\hline$M_{4}$ & 014 & 028 & 066 & 124 & .843 & 0.916 & 0.990 & 0.994 & .008 & 0.025 & .232 & 0.344 & .000 & 1.000 \\
\hline$L M_{5}$ & 008 & .026 & 082 & 164 & 0.667 & 0.789 & 0.964 & 0.984 & 0.012 & 0.032 & .114 & 0.186 & .000 & 1.000 \\
\hline$L M_{6}$ & 016 & 038 & 094 & 186 & 0.812 & 0.902 & 0.996 & 0.996 & 0.017 & 0.038 & .202 & 0.310 & .000 & 1.000 \\
\hline$L M_{7}$ & 0.020 & 0.048 & 694 & .778 & 0.759 & 0.854 & 1.000 & 1.000 & 0.032 & 0.058 & 0.160 & 0.252 & 1.000 & 1.000 \\
\hline$L M_{8}$ & & 0.052 & & .392 & 0.257 & 0.393 & 0.998 & 1.000 & 0.018 & 0.047 & 0.112 & 0.214 & 1.000 & 1.000 \\
\hline \multicolumn{15}{|c|}{ CM tests } \\
\hline$C M_{1}^{\perp}$ & 096 & .140 & .860 & .918 & 0.743 & 0.843 & 1.000 & 1.000 & 0.062 & 0.113 & 0.224 & 0.346 & 1.000 & 1.000 \\
\hline$C M_{2}^{1}$ & .082 & 0.148 & 838 & .896 & 0.701 & 0.808 & 1.000 & 1.000 & 0.063 & 0.113 & 0.250 & 0.374 & 1.000 & 1.000 \\
\hline$C M_{3}^{1}$ & 100 & .156 & 942 & 964 & 0.910 & 0.948 & 1.000 & 1.000 & 0.058 & 0.122 & 0.306 & 0.458 & 1.000 & 1.000 \\
\hline$C M_{4}^{1}$ & 0.090 & 0.150 & 0.872 & .916 & 0.887 & 0.941 & 1.000 & 1.000 & 0.058 & 0.132 & 0.324 & 0.470 & .000 & 1.000 \\
\hline$C M_{5}^{1}$ & 0.060 & 0.130 & 0.960 & .976 & 0.893 & 0.946 & 1.000 & 1.000 & 0.072 & 0.135 & 0.342 & 0.496 & 1.000 & 1.000 \\
\hline$C M_{6}^{1}$ & 0.062 & 0.128 & 0.936 & .964 & 0.843 & 0.908 & 1.000 & 1.000 & 0.070 & 0.147 & 0.326 & 0.492 & 0.998 & 0.998 \\
\hline$C M_{7}^{1}$ & .078 & .132 & 994 & .994 & 0.960 & 0.979 & 1.000 & 1.000 & 0.077 & 0.130 & 0.438 & 0.590 & 1.000 & 1.000 \\
\hline$C M_{8}^{1}$ & 0.074 & 0.134 & 968 & 982 & 0.935 & 0.964 & 1.000 & 1.000 & 0.080 & 0.142 & 0.422 & 0.558 & 1.000 & 1.000 \\
\hline$C M_{9}^{1}$ & 0.072 & 0.126 & 0.874 & .918 & 0.935 & 0.962 & 1.000 & 1.000 & 0.067 & 0.127 & 0.320 & 0.464 & 1.000 & 1.000 \\
\hline$C M_{10}^{1}$ & 0.068 & 0.124 & .866 & 914 & 0.881 & 0.946 & 1.000 & 1.000 & 0.067 & 0.132 & 0.312 & 0.446 & 1.000 & 1.000 \\
\hline$C M_{11}^{1}$ & 0.080 & 0.146 & 0.912 & 948 & 0.969 & 0.981 & 1.000 & 1.000 & 0.070 & 0.137 & 0.410 & 0.564 & 1.000 & 1.000 \\
\hline$C M_{12}^{1}$ & 0.084 & 0.134 & 0.856 & .910 & 0.950 & 0.977 & 1.000 & 1.000 & 0.077 & 0.140 & 0.412 & 0.528 & 1.000 & 1.000 \\
\hline$C M_{1}^{2}$ & 0.058 & 0.128 & 0.930 & 968 & 0.925 & 0.960 & 1.000 & 1.000 & 0.072 & 0.123 & 0.292 & 0.442 & 1.000 & 1.000 \\
\hline$C M_{2}^{2}$ & 0.064 & .126 & 924 & 950 & 0.845 & 0.923 & 1.000 & 1.000 & 0.077 & 0.120 & 0.310 & 0.448 & 1.000 & 1.000 \\
\hline$C M_{3}^{2}$ & 0.084 & 0.154 & .990 & 992 & 0.952 & 0.975 & 1.000 & 1.000 & 0.068 & 0.142 & 0.394 & 0.528 & 1.000 & 1.000 \\
\hline$C M_{4}^{2}$ & 0.082 & 0.144 & .972 & .982 & 0.927 & 0.964 & 1.000 & 1.000 & 0.072 & 0.152 & 0.402 & 0.528 & 1.000 & 1.000 \\
\hline$C M_{5}^{2}$ & 0.088 & .160 & 0.132 & 222 & 0.918 & 0.958 & 1.000 & 1.000 & 0.048 & 0.098 & 0.744 & 0.842 & 0.978 & 0.990 \\
\hline$C M_{6}^{2}$ & 0.086 & .178 & 0.072 & 136 & 0.435 & 0.579 & 0.908 & 0.950 & 0.048 & 0.095 & 0.566 & 0.714 & 0.618 & 0.738 \\
\hline$C M_{7}^{2}$ & 0.090 & 0.136 & 0.236 & 342 & 0.992 & 0.998 & 1.000 & 1.000 & 0.043 & 0.105 & 0.762 & 0.844 & 1.000 & 1.000 \\
\hline & 0.084 & 0.140 & 106 & .164 & 0.866 & 0.925 & 0.998 & 1.000 & 0.045 & 0.097 & 0.730 & 0.808 & 0.954 & 0.980 \\
\hline$M_{9}^{2}$ & 0.076 & .126 & 980 & 986 & 0.927 & 0.954 & 1.000 & 1.000 & 0.098 & 0.182 & 0.366 & 0.488 & 1.000 & 1.000 \\
\hline$C M_{10}^{2}$ & .068 & .144 & 946 & 970 & 0.843 & 0.910 & 1.000 & 1.000 & 0.105 & 0.190 & 0.366 & 0.474 & 1.000 & 1.000 \\
\hline$C M_{11}^{2}$ & 0.098 & 0.180 & 0.864 & 918 & 0.682 & 0.793 & 1.000 & 1.000 & 0.087 & 0.148 & 0.332 & 0.464 & 1.000 & 1.000 \\
\hline$C M_{12}^{2}$ & 0.094 & 0.170 & 0.782 & 0.856 & 0.644 & 0.753 & 1.000 & 1.000 & 0.085 & 0.158 & 0.346 & 0.448 & 1.000 & 1.000 \\
\hline \multicolumn{15}{|c|}{ F-tests against nonlinearites in the news response } \\
\hline$N L_{1}$ & .064 & .108 & .972 & 0.984 & 0.918 & 0.952 & 1.000 & 1.000 & 0.077 & 0.162 & 0.360 & & 1.000 & 1.000 \\
\hline & 0.040 & .104 & 972 & 986 & 960 & 0.979 & 1.000 & 1.000 & 0.072 & 0.1 & 0.422 & 0.550 & 1.000 & 1.000 \\
\hline & .056 & .118 & 0.670 & .788 & 0.533 & 0.653 & 1.000 & 1.000 & 0.082 & 0.168 & 0.270 & 0.392 & 1.000 & 1.000 \\
\hline$N L_{4}$ & & & 0.602 & & & & 1.000 & 1.000 & 0.067 & 0.127 & 0.292 & 0.412 & 1.000 & 1.000 \\
\hline \multicolumn{15}{|c|}{ ICM tests } \\
\hline$I C M_{1}$ & & & & & & & 0.378 & 0.4 & 08 & & 066 & & .198 & 0.274 \\
\hline $\mathrm{ICM}_{2}$ & 0.030 & 0.064 & .046 & 0.096 & 0.044 & 0.088 & & 0.436 & 0.003 & 0.023 & 0.074 & 0.166 & 0.150 & 0.228 \\
\hline $\mathrm{ICM}_{3}$ & 0.034 & 0.066 & 0.042 & 0.102 & 0.048 & 0.094 & 0.334 & 0.422 & 0.005 & 0.015 & 0.074 & 0.166 & 0.122 & 0.196 \\
\hline $\mathrm{ICM}_{4}$ & 0.038 & 0.084 & 0.044 & 0.110 & 0.046 & 0.109 & 0.296 & 0.430 & 0.003 & 0.022 & 0.078 & 0.172 & 0.136 & 0.204 \\
\hline
\end{tabular}




\section{A.2. Empirical Application to NYSE Financial Durations.}

Table 5: Descriptive statistics and Ljung-Box statistics of plain and seasonally adjusted trade durations and price durations for the AOL, Coca-Cola, Disney and GE stock traded at the NYSE. Data extracted from the TAQ data base, sample period from $02 / 01 / 01$ to $05 / 30 / 01$ for price durations and from $03 / 19 / 01$ to $03 / 30 / 01$ for trade durations.

\begin{tabular}{lcccccccc}
\hline & \multicolumn{4}{c}{ AOL } & \multicolumn{3}{c}{ Coca-Cola } \\
& trade durations & price durations & \multicolumn{2}{c}{ trade durations } & \multicolumn{2}{c}{$\begin{array}{c}\text { price durations } \\
\text { plain }\end{array}$} \\
& plain & adj. & plain & adj. & plain & adj. & adj. \\
\hline Obs & 20988 & 20988 & 10083 & 10083 & 15174 & 15174 & 12971 & 12971 \\
Mean & 11.121 & 1.000 & 237.936 & 1.000 & 15.302 & 1.000 & 183.659 & 1.000 \\
Std.dev. & 13.631 & 1.221 & 302.962 & 1.141 & 19.231 & 1.255 & 248.438 & 1.287 \\
LB(20) & 851 & 723 & 2809 & 2138 & 232 & 138 & 3166 & 2318 \\
\hline \hline & trade durations & price durations & trade durations & price durations \\
& plain & adj. & plain & adj. & plain & adj. & plain & adj. \\
\hline Obs & 16272 & 16272 & 9618 & 9618 & 25101 & 25101 & 16008 & 16008 \\
Mean & 14.335 & 1.000 & 248.000 & 1.000 & 8.881 & 1.000 & 150.497 & 0.999 \\
Std.dev. & 16.984 & 1.151 & 341.513 & 1.249 & 9.245 & 1.038 & 195.008 & 1.200 \\
LB(20) & 548 & 194 & 1842 & 823 & 875 & 803 & 8466 & 4827
\end{tabular}

Price durations for AOL stock based on $\$ 0.1$ midquote changes and for the Coca-Cola, Disney and GE stock based on $\$ 0.05$ midquote changes. Descriptive statistics of durations in seconds. 
Table 6: QML estimates of various types of ACD models for AOL trade durations and $\$ 0.100$ price durations. Data extracted from the TAQ database available from the NYSE, sample period from 03/19/01 to 03/30/01 for trade durations and $01 / 02 / 01$ to $05 / 30 / 01$ for price durations. Standard errors based on OPG estimates (in brackets). NPACD model estimated based on the category bounds $(0.1,0.2,0.5,1.0,1.5,2.0,3.0)$ with $\bar{\varepsilon}_{0}=1.0$. The threshold values for the three-regime TACD model are fixed exogenously to 0.25 and 1.50 . The estimates of the TACD model are shown in the same rows as those of the SPACD model.

\begin{tabular}{|c|c|c|c|c|c|c|c|c|c|c|c|c|c|c|}
\hline & $(1)$ & $(2)$ & $(3)$ & $(4)$ & $(5)$ & (6) & $(7)$ & $(1)$ & $(2)$ & (3) & $(4)$ & $(5)$ & $(6)$ & $(7)$ \\
\hline & $\mathrm{ACD}$ & LACD & BACD & EXACD & AGACD & DSPACD & TACD & ACD & LACD & BACD & EXACD & AGACD & DSPACD & T TACD \\
\hline \multirow[t]{2}{*}{$\omega$} & 0.009 & $-0.022-$ & -0.024 & -0.024 & -0.001 & -0.014 & -0.040 & 0.029 & -0.080 & -0.512 & -0.066 & 0.021 & 0.036 & -0.010 \\
\hline & $(0.001)$ & $(0.001)$ & $(0.007)$ & $(0.002)$ & $(0.012)$ & $(0.004)$ & $(0.015)$ & $(0.005)$ & $(0.005)$ & $(0.215)$ & $(0.006)$ & $(0.009)$ & $(0.014)$ & $(0.012)$ \\
\hline \multirow[t]{2}{*}{$\alpha_{1}$} & -0.005 & $-0.003-$ & -0.002 & -0.020 & -0.102 & & 0.181 & 0.129 & 0.106 & 0.848 & 0.190 & -0.233 & & 0.136 \\
\hline & $(0.005)$ & $(0.005)$ & $(0.007)$ & $(0.009)$ & $(0.023)$ & & $(0.134)$ & $(0.013)$ & $(0.011)$ & $(0.340)$ & $(0.014)$ & $(0.035)$ & & $(0.145)$ \\
\hline \multirow[t]{2}{*}{$\alpha_{2}$} & 0.030 & 0.025 & 0.036 & 0.038 & 0.185 & & -0.171 & -0.039 & -0.027 & -0.304 & -0.071 & 0.130 & & 0.228 \\
\hline & $(0.005)$ & $(0.005)$ & $(0.011)$ & $(0.009)$ & $(0.037)$ & & $(0.124)$ & $(0.013)$ & $(0.011)$ & $(0.137)$ & $(0.015)$ & $(0.030)$ & & $(0.134)$ \\
\hline \multirow[t]{2}{*}{$\beta_{1}$} & 0.967 & 0.991 & 0.990 & 0.989 & 0.935 & 0.981 & 1.030 & 0.883 & 0.967 & 0.968 & 0.967 & 0.968 & 0.972 & 0.835 \\
\hline & $(0.002)$ & $(0.001)$ & $(0.002)$ & $(0.002)$ & $(0.019)$ & $(0.002)$ & $(0.011)$ & $(0.010)$ & $(0.005)$ & $(0.005)$ & $(0.006)$ & $(0.005)$ & $(0.005)$ & ) $(0.016)$ \\
\hline \multirow[t]{2}{*}{$\delta_{1}$} & & & 1.585 & & 1.897 & & & & & $-^{*}$ & & $-^{*}$ & & \\
\hline & & & $(0.350)$ & & $(0.662)$ & & & & & $-*$ & & $-*$ & & \\
\hline \multirow[t]{2}{*}{$\delta_{2}$} & & & 1.055 & & 0.647 & & & & & 0.164 & & 1.000 & & \\
\hline & & & (0.077) & & $(0.074)$ & & & & & $(0.071)$ & & - & & \\
\hline$b$ & & & & & $\begin{array}{c}0.499 \\
(0.027)\end{array}$ & & & & & & & $\begin{array}{c}0.697 \\
(0.061)\end{array}$ & & \\
\hline \multirow[t]{2}{*}{$c_{1}$} & & & & 0.029 & 1.000 & & & & & & -0.161 & -1.172 & & \\
\hline & & & & $(0.012)$ & - & & & & & & $(0.020)$ & $(0.069)$ & & \\
\hline \multirow[t]{2}{*}{$c_{2}$} & & & & -0.022 & 0.061 & & & & & & 0.084 & -0.934 & & \\
\hline & & & & $(0.012)$ & $(0.144)$ & & & & & & $(0.020)$ & $(0.090)$ & & \\
\hline$\nu_{1}$ & & & & & $\begin{array}{c}0.091 \\
(0.021)\end{array}$ & & & & & & & $0.000^{* *}$ & & \\
\hline$\nu_{2}$ & & & & & $\begin{array}{c}-0.077 \\
(0.033)\end{array}$ & & & & & & & $0.000^{* *}$ & & \\
\hline \multirow[t]{2}{*}{$\alpha_{1}^{+}\left(\omega^{2}\right)$} & & & & & & 0.030 & 0.027 & & & & & & 0.035 & 0.052 \\
\hline & & & & & & $(0.010)$ & $(0.006)$ & & & & & & $(0.026)$ & $(0.014)$ \\
\hline \multirow[t]{2}{*}{$\alpha_{2}^{+}\left(\alpha_{1}^{2}\right)$} & & & & & & -0.017 & 0.029 & & & & & & -0.037 & 0.147 \\
\hline & & & & & & $(0.019)$ & $(0.020)$ & & & & & & $(0.053)$ & $(0.029)$ \\
\hline \multirow[t]{2}{*}{$\alpha_{3}^{+}\left(\alpha_{2}^{2}\right)$} & & & & & & 0.003 & -0.012 & & & & & & 0.059 & -0.047 \\
\hline & & & & & & $(0.016)$ & $(0.021)$ & & & & & & $(0.047)$ & $(0.029)$ \\
\hline \multirow[t]{2}{*}{$\alpha_{4}^{+}\left(\beta_{1}^{2}\right)$} & & & & & & -0.017 & 0.947 & & & & & & -0.027 & 0.870 \\
\hline & & & & & & $(0.007)$ & $(0.006)$ & & & & & & $(0.024)$ & $(0.018)$ \\
\hline \multirow[t]{2}{*}{$\alpha_{1}^{-}\left(\omega^{3}\right)$} & & & & & & -0.037 & 0.058 & & & & & & 0.245 & 0.081 \\
\hline & & & & & & $(0.014)$ & $(0.012)$ & & & & & & $(0.028)$ & $(0.032)$ \\
\hline \multirow[t]{2}{*}{$\alpha_{2}^{-}\left(\alpha_{1}^{3}\right)$} & & & & & & -0.076 & -0.006 & & & & & & 0.211 & 0.070 \\
\hline & & & & & & $(0.020)$ & $(0.006)$ & & & & & & $(0.057)$ & $(0.015)$ \\
\hline \multirow[t]{2}{*}{$\alpha_{3}^{-}\left(\alpha_{2}^{3}\right)$} & & & & & & 0.029 & 0.024 & & & & & & 0.463 & -0.023 \\
\hline & & & & & & $(0.048)$ & $(0.006)$ & & & & & & $(0.177)$ & $(0.012)$ \\
\hline$\alpha_{4}^{-}\left(\beta_{1}^{3}\right)$ & & & & & & -0.448 & 0.942 & & & & & & 0.064 & 0.920 \\
\hline & & & & & & (0.199) & $(0.012)$ & & & & & & $(0.410)$ & $(0.027)$ \\
\hline$a^{+}$ & & & & & & 0.090 & & & & & & & -0.366 & \\
\hline & & & & & & $(0.025)$ & & & & & & & $(0.040)$ & \\
\hline$a^{-}$ & & & & & & 0.002 & & & & & & & -0.008 & \\
\hline & & & & & & $(0.004)$ & & & & & & & $(0.009)$ & \\
\hline$\overline{O b s}$ & 20988 & 20988 & 20988 & 20988 & 20988 & 20988 & 20988 & 10083 & 10083 & 10083 & 10083 & 10083 & 10083 & 10083 \\
\hline LL & -20576 & -20578 & -20572 & -20571 & -20540 & -20545 & -20548 & -9385 & -9395 & -9342 & -9344 & -9340 & -9336 & -9343 \\
\hline $\mathrm{BIC}$ & -20596 & -20598 & -20602 & -20601 & -20589 & -20605 & -20608 & -9404 & -9413 & -9365 & -9372 & -9372 & -9391 & -9398 \\
\hline$\overline{\hat{\varepsilon}}_{i}$ & 1.000 & 1.001 & 1.001 & 1.002 & 1.001 & 1.001 & 1.001 & 1.005 & 1.005 & 1.005 & 1.008 & 1.004 & 1.006 & 1.005 \\
\hline SD & 1.164 & 1.164 & 1.162 & 1.161 & 1.152 & 1.154 & 1.155 & 1.051 & 1.051 & 1.047 & 1.047 & 1.043 & 1.045 & 1.048 \\
\hline LB & 32.179 & 33.222 & 31.937 & 32.915 & 29.072 & 29.640 & 30.446 & 10.056 & 9.867 & 13.955 & 10.623 & 11.453 & 12.427 & 11.896 \\
\hline
\end{tabular}

Diagnostics: Log Likelihood (LL), Bayes Information Criterion (BIC), mean $\left(\overline{\hat{\varepsilon}}_{i}\right)$, standard devation (SD) and Ljung-Box statistic with respect to 20 lags (LB) of ACD residuals.

${ }^{*}$ : Estimation based on logarithmic model. ${ }^{* *}$ : Parameters set to zero. 
Table 7: P-values of the different types of diagnostic tests (see Table 2) for the estimates based on AOL trade durations and price durations (see Table 6).

\begin{tabular}{|c|c|c|c|c|c|c|c|c|c|c|c|c|c|c|}
\hline & \multicolumn{7}{|c|}{ Trade durations } & \multicolumn{6}{|c|}{ Price durations } & \multirow[b]{2}{*}{ (7) } \\
\hline & (1) & (2) & $(3)$ & $(4)$ & $(5)$ & (6) & (7) & (1) & $(2)$ & $(3)$ & $(4)$ & (5) & (6) & \\
\hline \multicolumn{15}{|c|}{ LM tests } \\
\hline$L M_{1}$ & 0.224 & 0.521 & 0.604 & 0.958 & 0.921 & 1.000 & 0.548 & 0.000 & 0.000 & 0.551 & 0.724 & 1.000 & 0.999 & 0.996 \\
\hline$L M_{2}$ & 0.086 & 0.116 & 0.329 & 0.381 & 0.709 & 0.965 & 0.323 & 0.000 & 0.000 & 0.020 & 0.038 & 0.190 & 0.453 & .662 \\
\hline$L M_{3}$ & 0.740 & 0.748 & 0.658 & 1.000 & 0.975 & 0.999 & 0.965 & .000 & 0.000 & 0.774 & 0.329 & 0.526 & 0.854 & .908 \\
\hline $\mathrm{LM}_{4}$ & 0.211 & 0.225 & 0.325 & 0.530 & 0.796 & 0.941 & 0.736 & .000 & 0.000 & 0.378 & 0.335 & 0.546 & 0.776 & 0.914 \\
\hline$L M_{5}$ & 0.402 & 0.382 & 0.713 & 0.629 & 0.932 & 0.986 & 0.716 & 0.000 & 0.000 & 0.218 & 0.162 & 0.296 & 0.690 & 0.135 \\
\hline$L M_{6}$ & 0.135 & 0.140 & 0.237 & 0.227 & 0.646 & 0.744 & 0.454 & .000 & 0.000 & 0.033 & 0.024 & 0.069 & 0.269 & 0.092 \\
\hline$L M_{7}$ & 012 & 003 & 0.013 & 0.004 & 0.081 & 0.309 & 0.052 & .000 & 0.000 & 0.093 & 0.006 & 0.069 & 0.551 & 0.385 \\
\hline$L M_{8}$ & 048 & 0.026 & 0.047 & 0.097 & 0.209 & 581 & 0.428 & 0.000 & 0.000 & 0.713 & 0.311 & 0.660 & 0.923 & 0.962 \\
\hline \multicolumn{15}{|c|}{ CM tests } \\
\hline$C M_{1}^{1}$ & 0.038 & 0.106 & 0.168 & 0.155 & 0.466 & 0.156 & 0.137 & 0.000 & 0.000 & 0.098 & 0.051 & 0.040 & 0.055 & 0.001 \\
\hline$C M_{2}^{1}$ & 0.030 & 106 & 0.260 & 0.172 & 0.584 & 0.211 & 0.141 & .000 & 0.000 & 0.394 & 0.116 & 0.068 & 0.076 & 0.002 \\
\hline$C M_{3}^{1}$ & 0.009 & 0.012 & 0.036 & 0.006 & 0.065 & 0.040 & 0.048 & .000 & 0.000 & 0.097 & 0.262 & 0.225 & 0.236 & 0.013 \\
\hline$C M_{4}^{1}$ & 0.009 & 0.011 & 0.052 & 0.006 & 0.060 & 0.039 & 0.040 & 0.000 & 0.000 & 0.290 & 0.480 & 0.361 & 0.355 & 0.031 \\
\hline$C M_{5}^{1}$ & 0.026 & 0.046 & 0.085 & 0.018 & 0.204 & 0.211 & 0.011 & 0.000 & 0.000 & 0.045 & 0.025 & 0.052 & 0.017 & 0.000 \\
\hline$C M_{6}^{1}$ & 0.024 & 0.067 & 0.113 & 0.104 & 0.339 & 0.302 & 0.035 & .000 & 0.000 & 0.149 & 0.063 & 0.081 & 0.022 & 0.001 \\
\hline$C M_{7}^{1}$ & 0.012 & 0.014 & 0.060 & 0.005 & 0.103 & 0.065 & 0.010 & .000 & 0.000 & 0.014 & 0.024 & 0.027 & 0.012 & 0.000 \\
\hline$C M_{8}^{1}$ & 0.007 & 0.009 & 0.059 & 0.007 & 0.108 & 0.053 & 0.020 & .000 & 0.000 & 0.118 & 0.095 & 0.065 & 0.026 & 0.003 \\
\hline$C M_{9}^{1}$ & 0.041 & 0.072 & 0.135 & 0.108 & 0.293 & 0.218 & 0.041 & 0.000 & 0.000 & 0.059 & 0.039 & 0.054 & 0.027 & 0.000 \\
\hline$C M_{10}^{1}$ & 0.029 & 0.080 & 0.160 & 0.161 & 0.395 & 0.282 & 0.069 & 0.000 & 0.000 & 0.183 & 0.082 & 0.098 & 0.041 & 0.001 \\
\hline$C M_{11}^{1}$ & 0.011 & 0.016 & 0.059 & 0.020 & 0.147 & 0.083 & 0.030 & 0.000 & 0.000 & 0.132 & 0.187 & 0.237 & 0.106 & 0.002 \\
\hline$C M_{12}^{1}$ & 0.004 & 0.007 & 0.041 & 0.009 & 0.111 & 0.053 & 0.027 & 0.000 & 0.000 & 0.412 & 0.328 & 0.286 & 0.130 & 0.012 \\
\hline$C M_{1}^{2}$ & 0.071 & 0.104 & 0.177 & 0.051 & 0.339 & 0.313 & 0.032 & 0.000 & 0.000 & 0.085 & 0.070 & 0.140 & 0.049 & 0.001 \\
\hline$C M_{2}^{2}$ & 0.075 & 0.175 & 0.192 & 0.257 & 0.581 & 0.457 & 0.087 & 0.000 & 0.000 & 0.225 & 0.159 & 0.216 & 0.072 & 0.003 \\
\hline$C M_{3}^{2}$ & 0.009 & 0.014 & 0.029 & 0.002 & 0.063 & 0.014 & 0.011 & 0.000 & 0.000 & 0.042 & 0.053 & 0.061 & 0.038 & 0.001 \\
\hline$C M_{4}^{2}$ & 0.005 & 0.010 & 0.033 & 0.004 & 0.060 & 0.010 & 0.015 & 0.000 & 0.000 & 0.136 & 0.065 & 0.059 & 0.046 & 0.006 \\
\hline$C M_{5}^{2}$ & 0.017 & 0.004 & 0.002 & 0.002 & 0.010 & 0.019 & 0.020 & 0.991 & 0.804 & 0.308 & 0.407 & 0.485 & 0.223 & 0.186 \\
\hline$C M_{6}^{2}$ & 0.129 & 0.054 & 0.013 & 0.048 & 0.060 & 0.084 & 0.124 & 0.841 & 0.981 & 0.836 & 0.766 & 0.775 & 0.601 & 0.259 \\
\hline$C M_{7}^{2}$ & 0.001 & 0.001 & 0.001 & 0.000 & 0.003 & 0.005 & 0.004 & 0.893 & 0.694 & 0.101 & 0.285 & 0.474 & 0.156 & 0.511 \\
\hline$C M_{8}^{2}$ & 0.001 & 0.001 & 0.001 & 0.000 & 0.003 & 0.005 & 0.004 & 0.893 & 0.694 & 0.101 & 0.285 & 0.474 & 0.156 & 0.511 \\
\hline$C M_{9}^{2}$ & 0.012 & 0.002 & 0.005 & 0.000 & 0.004 & 0.013 & 0.002 & 0.000 & 0.000 & 0.040 & 0.000 & 0.006 & 0.036 & 0.007 \\
\hline$C M_{10}^{2}$ & 0.032 & 0.006 & 0.007 & 0.003 & 0.014 & 0.021 & 0.006 & 0.000 & 0.000 & 0.093 & 0.004 & 0.028 & 0.092 & 0.002 \\
\hline$C M_{11}^{2}$ & 0.020 & 0.009 & 0.008 & 0.021 & 0.015 & 0.054 & 0.036 & 0.000 & 0.000 & 0.171 & 0.023 & 0.057 & 0.137 & 0.037 \\
\hline$C M_{12}^{2}$ & 0.022 & 0.010 & 0.011 & 0.023 & 0.031 & 0.055 & 0.065 & 0.000 & 0.000 & 0.161 & 0.042 & 0.084 & 0.177 & 0.004 \\
\hline \multicolumn{15}{|c|}{ F-tests against nonlinearites in the news response } \\
\hline$N L_{1}$ & 0.007 & 0.006 & 0.013 & 0.006 & 0.013 & 0.008 & $\overline{0.016}$ & 0.000 & 0.000 & 0.188 & 0.090 & 0.491 & 0.788 & 0.254 \\
\hline$N L_{2}$ & 0.005 & 0.001 & 0.001 & 0.001 & 0.010 & 0.020 & 0.008 & 0.000 & 0.000 & 0.090 & 0.002 & 0.032 & 0.156 & 0.076 \\
\hline$N L_{3}$ & 0.013 & 0.009 & 0.007 & 0.025 & 0.031 & 0.041 & 0.086 & 0.000 & 0.000 & 0.314 & 0.151 & 0.300 & 0.412 & 0.410 \\
\hline$N L_{4}$ & 0.016 & 0.009 & 0.008 & 0.013 & 0.035 & 0.047 & 0.076 & 0.000 & 0.000 & 0.512 & 0.163 & 0.301 & 0.513 & 0.696 \\
\hline \multicolumn{15}{|c|}{ ICM tests } \\
\hline$I C M_{1}$ & 0.540 & 0.480 & 0.390 & 0.270 & 0.600 & 0.330 & 0.430 & 0.090 & 0.060 & 0.610 & 0.610 & 0.610 & 0.780 & 0.540 \\
\hline$I C M_{2}$ & 0.510 & 0.380 & 0.380 & 0.170 & 0.530 & 0.270 & 0.450 & 0.080 & 0.040 & 0.650 & 0.700 & 0.630 & 0.610 & 0.480 \\
\hline$I C M_{3}$ & 0.480 & 0.420 & 0.360 & 0.230 & 0.580 & 0.350 & 0.400 & 0.130 & 0.020 & 0.550 & 0.680 & 0.650 & 0.660 & 0.430 \\
\hline
\end{tabular}


Table 8: QML estimates of various types of ACD models for Coca-Cola trade durations and $\$ 0.05$ price durations. Data extracted from the TAQ database available from the NYSE, sample period from 03/19/01 to 03/30/01 for intertrade durations and 01/02/01 to $05 / 30 / 01$ for price durations. Standard errors based on OPG estimates (in brackets). SPACD model estimated based on the category bounds $(0.1,0.2,0.5,1.0,1.5,2.0,3.0)$ with $\bar{\varepsilon}_{0}=1.0$. The threshold values for the three-regime TACD model are fixed exogenously to 0.25 and 1.50 . The estimates of the TACD model are shown in the same rows as those of the SPACD model.

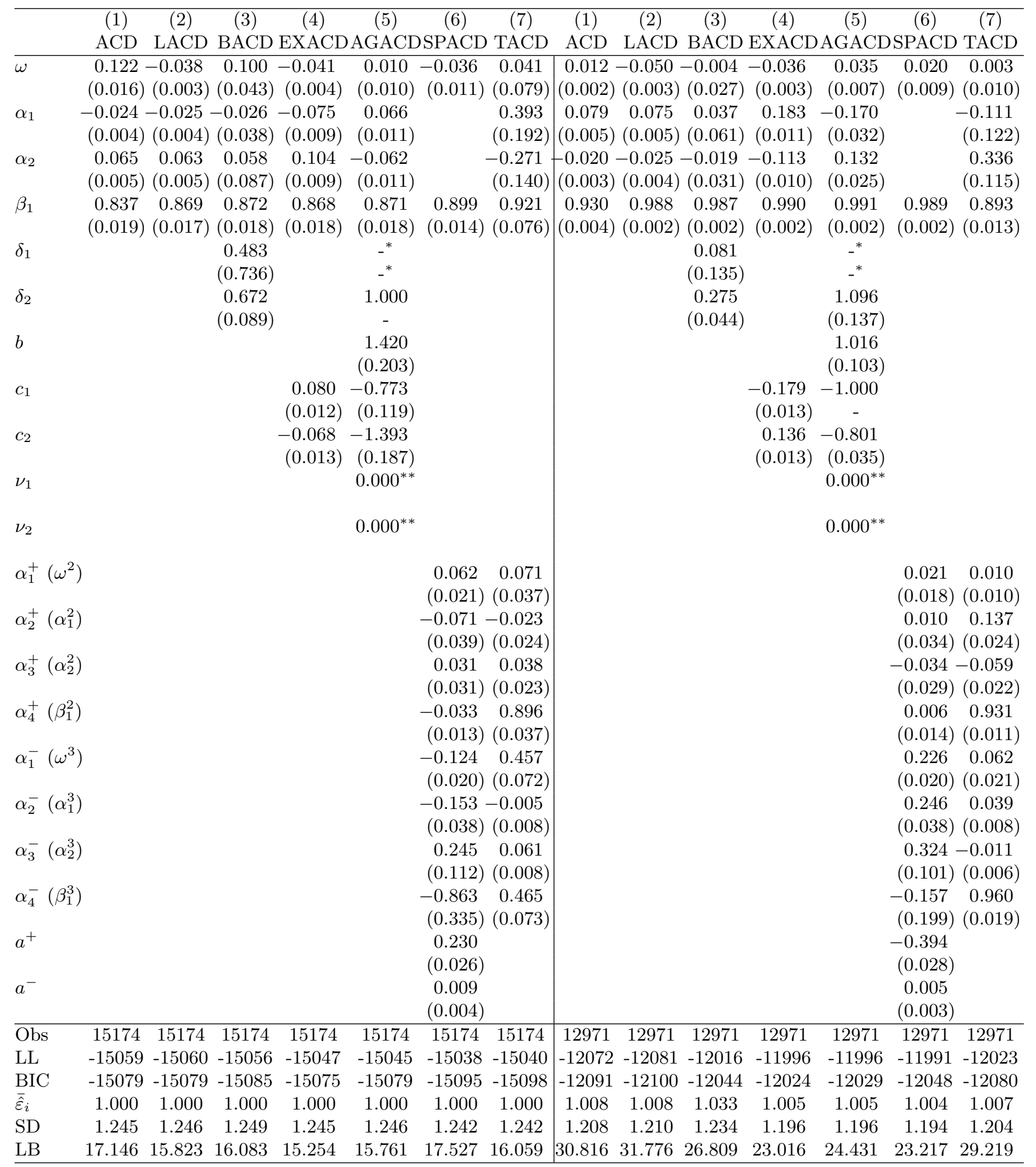

Diagnostics: Log Likelihood (LL), Bayes Information Criterion (BIC), mean $\left(\overline{\hat{\varepsilon}}_{i}\right)$, standard devation (SD) and Ljung-Box(20) statistic with respect to 20 lags (LB) of ACD residuals.

*: Estimation based on logarithmic model. ${ }^{* *}$ : Parameters set to zero. 
Table 9: P-values of the different types of diagnostic tests (see Table 2) for the estimates based on Coca-Cola trade durations and price durations (see Table 8).

\begin{tabular}{|c|c|c|c|c|c|c|c|c|c|c|c|c|c|c|}
\hline & \multicolumn{7}{|c|}{ Trade durations } & \multicolumn{6}{|c|}{ Price durations } & \multirow[b]{2}{*}{$(7)$} \\
\hline & (1) & (2) & $(3)$ & $(4)$ & $(5)$ & (6) & (7) & (1) & $(2)$ & (3) & $(4)$ & (5) & (6) & \\
\hline \multicolumn{15}{|c|}{ LM tests } \\
\hline$L M_{1}$ & 0.015 & 0.012 & 0.152 & 1.000 & 0.998 & 1.000 & 0.704 & 0.000 & 0.000 & 0.269 & 0.216 & 0.373 & 0.665 & 0.027 \\
\hline$L M_{2}$ & .026 & 0.025 & 0.177 & 0.998 & 0.995 & 1.000 & 0.828 & 0.000 & 0.000 & 0.121 & 0.179 & 0.298 & 0.587 & 0.010 \\
\hline$L M_{3}$ & 019 & 0.020 & 0.249 & 0.994 & 0.999 & 1.000 & 0.728 & .000 & 0.000 & 0.206 & 0.233 & 0.363 & 0.647 & 0.043 \\
\hline$L M_{4}$ & 0.056 & 0.033 & 0.239 & 0.981 & 0.996 & 1.000 & 0.823 & 1.000 & 0.000 & 0.081 & 0.160 & 0.239 & 0.519 & 0.014 \\
\hline$L M_{5}$ & 0.010 & 0.007 & 0.151 & 0.900 & 0.940 & 0.995 & 0.754 & 0.000 & 0.000 & 0.351 & 0.384 & 0.530 & 0.760 & 0.030 \\
\hline$L M_{6}$ & 0.025 & 0.012 & 0.225 & 0.867 & 0.949 & 0.999 & 0.908 & 0.000 & 0.000 & 0.153 & 0.156 & 0.222 & 0.381 & 0.012 \\
\hline$L M_{7}$ & 142 & 0.130 & 0.391 & 0.973 & 0.993 & 0.997 & 0.758 & .000 & 0.000 & 0.411 & 0.485 & 0.478 & 0.660 & 0.061 \\
\hline$L M_{8}$ & 051 & 0.038 & 0.129 & 0.819 & 0.874 & .953 & 0.432 & 0.000 & 0.000 & 0.102 & 0.128 & 0.187 & 0.452 & 0.006 \\
\hline \multicolumn{15}{|c|}{ CM tests } \\
\hline$C M_{1}^{1}$ & .000 & 0.000 & 0.000 & 0.101 & 0.068 & 0.019 & 0.016 & 0.000 & 0.000 & 0.013 & 0.024 & 0.037 & 0.019 & 0.000 \\
\hline$C M_{2}^{1}$ & 000 & 0.000 & 0.000 & 0.038 & 0.024 & 0.013 & 0.020 & .000 & 0.000 & 0.027 & 0.066 & 0.084 & 0.037 & .000 \\
\hline$C M_{3}^{1}$ & 0.000 & 0.000 & 0.000 & 0.010 & 0.012 & 0.002 & 0.011 & 0.000 & 0.000 & 0.024 & 0.019 & 0.023 & 0.021 & 0.000 \\
\hline$C M_{4}^{1}$ & 0.000 & 0.000 & 0.000 & 0.003 & 0.003 & 0.001 & 0.013 & 0.000 & 0.000 & 0.049 & 0.040 & 0.044 & 0.035 & 0.000 \\
\hline$C M_{5}^{1}$ & 0.000 & 0.000 & 0.001 & 0.212 & 0.185 & 0.216 & 0.013 & 0.000 & 0.000 & 0.031 & 0.012 & 0.021 & 0.005 & 0.000 \\
\hline$C M_{6}^{1}$ & 000 & 0.000 & 0.000 & 0.058 & 0.026 & 0.180 & 0.029 & 0.000 & 0.000 & 0.303 & 0.053 & 0.083 & 0.020 & 0.000 \\
\hline$C M_{7}^{1}$ & 0.001 & 0.000 & 0.001 & 0.045 & .087 & 0.397 & 0.075 & .000 & 0.000 & 0.004 & 0.004 & 0.007 & 0.001 & 0.000 \\
\hline$C M_{8}^{1}$ & 0.001 & 0.000 & 0.000 & 0.052 & 0.036 & 0.326 & 0.113 & 0.000 & 0.000 & 0.067 & 0.028 & 0.059 & 0.010 & 0.000 \\
\hline$C M_{9}^{1}$ & 0.001 & 0.000 & 0.003 & 0.240 & 0.231 & 0.243 & 0.019 & 0.000 & 0.000 & 0.025 & 0.011 & 0.020 & 0.006 & 0.000 \\
\hline$C M_{10}^{1}$ & 0.001 & 0.000 & 0.001 & 0.046 & 0.029 & 0.192 & 0.040 & 0.000 & 0.000 & 0.145 & 0.043 & 0.068 & 0.018 & 0.000 \\
\hline$C M_{11}^{1}$ & 0.002 & 0.000 & 0.002 & 0.132 & 0.175 & 0.456 & 0.080 & 0.000 & 0.000 & 0.006 & 0.002 & 0.004 & 0.001 & 0.000 \\
\hline$C M_{12}^{1}$ & 0.001 & 0.000 & 0.001 & 0.093 & 0.069 & 0.374 & 0.123 & 0.000 & 0.000 & 0.036 & 0.009 & 0.020 & 0.004 & 0.000 \\
\hline$C M_{1}^{2}$ & 0.001 & 0.000 & 0.001 & 0.065 & 0.080 & 0.302 & 0.041 & 0.000 & 0.000 & 0.046 & 0.039 & 0.061 & 0.020 & 0.000 \\
\hline$C M_{2}^{2}$ & 0.001 & 0.000 & 0.000 & 0.064 & 0.030 & 0.286 & 0.088 & 0.000 & 0.000 & 0.287 & 0.083 & 0.135 & 0.059 & 0.000 \\
\hline$C M_{3}^{2}$ & 0.005 & 0.000 & 0.003 & 0.103 & 0.175 & 0.682 & 0.263 & 0.000 & 0.000 & 0.006 & 0.006 & 0.008 & 0.003 & 0.000 \\
\hline$C M_{4}^{2}$ & 0.005 & 0.001 & 0.001 & 0.157 & 0.112 & 0.626 & 0.367 & 0.000 & 0.000 & 0.060 & 0.040 & 0.067 & 0.027 & 0.000 \\
\hline$C M_{5}^{2}$ & 0.021 & 0.035 & 0.598 & 0.072 & 0.095 & 0.131 & 0.222 & 0.072 & 0.043 & 0.036 & 0.134 & 0.111 & 0.109 & 0.014 \\
\hline$C M_{6}^{2}$ & 0.005 & 0.001 & 0.445 & 0.019 & 0.029 & 0.098 & 0.148 & 0.477 & 0.513 & 0.091 & 0.395 & 0.319 & 0.327 & 0.044 \\
\hline$C M_{7}^{2}$ & 0.254 & 0.172 & 0.381 & 0.259 & 0.230 & 0.249 & 0.414 & 0.009 & 0.008 & 0.017 & 0.044 & 0.041 & 0.040 & 0.004 \\
\hline$C M_{8}^{2}$ & 0.254 & 0.172 & 0.381 & 0.259 & 0.230 & 0.249 & 0.414 & 0.009 & 0.008 & 0.017 & 0.044 & 0.041 & 0.040 & 0.004 \\
\hline$C M_{9}^{2}$ & 0.033 & 0.029 & 0.068 & 0.792 & 0.896 & 0.715 & 0.149 & 0.000 & 0.000 & 0.045 & 0.096 & 0.076 & 0.022 & 0.000 \\
\hline$C M_{10}^{2}$ & 0.028 & 0.027 & 0.047 & 0.711 & 0.868 & 0.621 & 0.209 & 0.000 & 0.000 & 0.080 & 0.283 & 0.259 & 0.116 & 0.001 \\
\hline$C M_{11}^{2}$ & 0.009 & 0.005 & 0.007 & 0.353 & 0.333 & 0.265 & 0.038 & 0.000 & 0.000 & 0.006 & 0.008 & 0.006 & 0.011 & 0.000 \\
\hline$C M_{12}^{2}$ & 0.010 & 0.006 & 0.005 & 0.327 & 0.291 & 0.228 & 0.117 & 0.000 & 0.000 & 0.038 & 0.089 & 0.063 & 0.104 & 0.000 \\
\hline \multicolumn{15}{|c|}{ F-tests against nonlinearites in the news response } \\
\hline$N L_{1}$ & .011 & 0.007 & 0.020 & 0.534 & 0.786 & 0.299 & 0.288 & 0.000 & 0.000 & 0.053 & 0.367 & 0.236 & 0.181 & 0.001 \\
\hline$N L_{2}$ & 0.060 & 0.056 & 0.094 & 0.766 & 0.904 & 0.786 & 0.462 & 0.000 & 0.000 & 0.115 & 0.308 & 0.159 & 0.168 & 0.015 \\
\hline$N L_{3}$ & 0.008 & 0.009 & 0.062 & 0.237 & 0.331 & 0.187 & 0.140 & 0.000 & 0.000 & 0.108 & 0.110 & 0.093 & 0.147 & 0.000 \\
\hline$N L_{4}$ & 0.050 & 0.063 & 0.176 & 0.540 & 0.597 & 0.521 & 0.429 & 0.000 & 0.000 & 0.074 & 0.038 & 0.026 & 0.068 & 0.000 \\
\hline \multicolumn{15}{|c|}{ ICM tests } \\
\hline$I C M_{1}$ & 0.950 & 0.900 & 0.860 & 0.240 & 0.240 & 0.480 & 0.430 & 0.840 & 0.970 & 0.660 & 0.410 & 0.340 & 0.280 & 0.470 \\
\hline $\mathrm{ICM}_{2}$ & 0.700 & 0.760 & 0.650 & 0.310 & 0.160 & 0.360 & 0.450 & 0.830 & 0.940 & 0.680 & 0.420 & 0.330 & 0.290 & 0.530 \\
\hline$I C M_{3}$ & 0.670 & 0.650 & 0.640 & 0.190 & 0.110 & 0.270 & 0.400 & 0.900 & 0.940 & 0.650 & 0.490 & 0.410 & 0.460 & 0.460 \\
\hline
\end{tabular}


Table 10: QML estimates of various types of ACD models for Disney trade durations and $\$ 0.05$ price durations. Data extracted from the TAQ database available from the NYSE, sample period from 03/19/01 to 03/30/01 for trade durations and $01 / 02 / 01$ to $05 / 30 / 01$ for price durations. Standard errors based on OPG estimates (in brackets). NPACD model estimated based on the category bounds $(0.1,0.2,0.5,1.0,1.5,2.0,3.0)$ with $\bar{\varepsilon}_{0}=1.0$. The threshold values for the three-regime TACD model are fixed exogenously to 0.25 and 1.50 . The estimates of the TACD model are shown in the same rows as those of the SPACD model.

\begin{tabular}{|c|c|c|c|c|c|c|c|c|c|c|c|c|c|c|}
\hline & $(1)$ & $(2)$ & $(3)$ & $(4)$ & $(5)$ & $(6)$ & $(7)$ & (1) & $(2)$ & $(3)$ & (4) & $(5)$ & $(6)$ & $(7)$ \\
\hline & ACD & LACD & BACD & EXACD & AGACD & SPACD & TACD & $\mathrm{ACD}$ & LACD & $\mathrm{BACD}$ & EXACD & AGACD & DSPACD & D TACD \\
\hline \multirow[t]{2}{*}{$\omega$} & 0.034 & -0.023 & -0.065 & -0.023 & -0.026 & 0.010 & 0.021 & $0.055-$ & -0.045 & -0.038 & -0.032 & -0.046 & 0.040 & -0.006 \\
\hline & $(0.006)$ & $(0.003)$ & $(0.028)$ & $(0.003)$ & $(0.017)$ & $(0.007)$ & $(0.036)$ & $(0.010)$ & $(0.008)$ & $(0.018)$ & $(0.005)$ & $(0.025)$ & $(0.011)$ & ) $(0.006)$ \\
\hline \multirow[t]{2}{*}{$\alpha_{1}$} & 0.018 & 0.017 & 0.076 & 0.038 & 0.070 & & 0.045 & 0.170 & 0.136 & 0.265 & 0.210 & -0.125 & & 0.563 \\
\hline & (0.006) & $(0.006)$ & $(0.031)$ & $(0.011)$ & $(0.052)$ & & $(0.043)$ & $(0.011)$ & $(0.008)$ & $(0.066)$ & $(0.012)$ & $(0.125)$ & & $(0.164)$ \\
\hline \multirow[t]{2}{*}{$\alpha_{2}$} & 0.007 & 0.006 & 0.042 & -0.009 & -0.037 & & 0.184 & $-0.089-$ & -0.092 & -0.184 & -0.147 & 0.056 & & -0.351 \\
\hline & $(0.006)$ & $(0.006)$ & $(0.029)$ & $(0.011)$ & $(0.046)$ & & $(0.060)$ & $(0.013)$ & $(0.009)$ & $(0.048)$ & $(0.012)$ & $(0.100)$ & & $(0.178)$ \\
\hline \multirow[t]{2}{*}{$\beta_{1}$} & 0.941 & 0.970 & 0.954 & 0.968 & 0.916 & 0.969 & 0.915 & 0.983 & 1.291 & 1.296 & 1.302 & 1.429 & 1.245 & 0.959 \\
\hline & (0.008) & $(0.006)$ & $(0.008)$ & $(0.006)$ & $(0.047)$ & $(0.006)$ & $(0.044)$ & $(0.099)$ & $(0.085)$ & $(0.069)$ & $(0.070)$ & $(0.165)$ & $(0.071)$ & ) $(0.064)$ \\
\hline \multirow[t]{2}{*}{$\beta_{2}$} & & & & & & & & -0.116 & -0.323 & -0.329 & -0.329 & -0.389 & -0.277 & -0.055 \\
\hline & & & & & & & & $(0.080)$ & $(0.080)$ & $(0.065)$ & $(0.066)$ & $(0.145)$ & $(0.067)$ & (0.053) \\
\hline \multirow[t]{2}{*}{$\delta_{1}$} & & & 3.268 & & 4.996 & & & & & 0.518 & & 0.047 & & \\
\hline & & & $(0.966)$ & & $(2.335)$ & & & & & $(0.138)$ & & 0.328 & & \\
\hline \multirow[t]{2}{*}{$\delta_{2}$} & & & 0.881 & & 0.895 & & & & & 0.339 & & 0.355 & & \\
\hline & & & $(0.126)$ & & (0.148) & & & & & $(0.052)$ & & 0.052 & & \\
\hline \multirow[t]{2}{*}{$b$} & & & & & 0.362 & & & & & & & 0.021 & & \\
\hline & & & & & $(0.150)$ & & & & & & & (0.008) & & \\
\hline \multirow[t]{2}{*}{$c_{1}$} & & & & -0.034 & 1.000 & & & & & & -0.134 & 1.000 & & \\
\hline & & & & $(0.014)$ & - & & & & & & $(0.016)$ & - & & \\
\hline \multirow[t]{2}{*}{$c_{2}$} & & & & 0.026 & 1.000 & & & & & & 0.092 & 1.000 & & \\
\hline & & & & $(0.014)$ & - & & & & & & $(0.015)$ & - & & \\
\hline$\nu_{1}$ & & & & & $\begin{array}{r}-0.003 \\
(0.035)\end{array}$ & & & & & & & $\begin{array}{c}0.142 \\
(0.062)\end{array}$ & & \\
\hline$\nu_{2}$ & & & & & $\begin{array}{c}0.065 \\
(0.035)\end{array}$ & & & & & & & -0.068 & & \\
\hline \multirow{2}{*}{$\alpha_{1}^{+}\left(\omega^{2}\right)$} & & & & & & 0.012 & 0.007 & & & & & & -0.018 & 0.036 \\
\hline & & & & & & $(0.014)$ & $(0.018)$ & & & & & & $(0.021)$ & $(0.018)$ \\
\hline \multirow[t]{2}{*}{$\alpha_{2}^{+}\left(\alpha_{1}^{2}\right)$} & & & & & & -0.034 & 0.019 & & & & & & 0.170 & 0.139 \\
\hline & & & & & & $(0.027)$ & $(0.019)$ & & & & & & $(0.041)$ & $(0.034)$ \\
\hline \multirow[t]{2}{*}{$\alpha_{3}^{+}\left(\alpha_{2}^{2}\right)$} & & & & & & 0.041 & 0.034 & & & & & & -0.112 & -0.038 \\
\hline & & & & & & $(0.023)$ & $(0.021)$ & & & & & & $(0.035)$ & ) (0.039) \\
\hline \multirow{2}{*}{$\alpha_{4}^{+}\left(\beta_{1}^{2}\right)$} & & & & & & -0.021 & 0.945 & & & & & & 0.039 & 1.109 \\
\hline & & & & & & $(0.010)$ & $(0.019)$ & & & & & & $(0.015)$ & $(0.074)$ \\
\hline \multirow[t]{2}{*}{$\alpha_{1}^{-}\left(\beta_{2}^{2}\right)$} & & & & & & 0.069 & & & & & & & 0.286 & -0.220 \\
\hline & & & & & & $(0.019)$ & & & & & & & $(0.023)$ & $(0.056)$ \\
\hline$\alpha_{2}^{-}\left(\omega^{3}\right)$ & & & & & & 0.019 & 0.156 & & & & & & 0.193 & 0.161 \\
\hline & & & & & & $(0.028)$ & $(0.046)$ & & & & & & $(0.040)$ & $(0.046)$ \\
\hline$\alpha_{3}^{-}\left(\alpha_{1}^{3}\right)$ & & & & & & -0.115 & 0.006 & & & & & & 0.380 & 0.095 \\
\hline & & & & & & $(0.086)$ & $(0.008)$ & & & & & & $(0.113)$ & ) (0.016) \\
\hline$\alpha_{4}^{-}\left(\alpha_{2}^{3}\right)$ & & & & & & 1.057 & 0.017 & & & & & & -0.073 & -0.063 \\
\hline & & & & & & $(0.354)$ & $(0.008)$ & & & & & & $(0.222)$ & $(0.014)$ \\
\hline$a^{+}\left(\beta_{1}^{3}\right)$ & & & & & & -0.082 & 0.831 & & & & & & -0.440 & 1.091 \\
\hline & & & & & & $(0.031)$ & $(0.043)$ & & & & & & $(0.031)$ & ) $(0.105)$ \\
\hline$a^{-}\left(\beta_{2}^{3}\right)$ & & & & & & 0.006 & & & & & & & -0.036 & -0.235 \\
\hline & & & & & & $(0.004)$ & & & & & & & $(0.006)$ & )$(0.081)$ \\
\hline$\overline{\mathrm{Obs}}$ & 16272 & 16272 & 16272 & 16272 & 16272 & 16272 & 16272 & 9618 & 9618 & 9618 & 9618 & 9618 & 9618 & 9618 \\
\hline LL & -16147 & -16147 & -16141 & -16144 & -16140 & -16138 & -16140 & -9039 & -9048 & -8986 & -8999 & -8982 & -8986 & -8984 \\
\hline $\mathrm{BIC}$ & -16166 & -16166 & -16170 & -16173 & -16183 & -16197 & -16198 & -9062 & -9071 & -9018 & -9031 & -9028 & -9046 & -9053 \\
\hline$\overline{\hat{\varepsilon}}_{i}$ & 1.002 & 1.001 & 1.001 & 1.001 & 1.001 & 0.999 & 1.001 & 1.004 & 1.004 & 1.004 & 1.003 & 1.004 & 0.998 & 1.004 \\
\hline SD & 1.135 & 1.134 & 1.134 & 1.135 & 1.133 & 1.131 & 1.135 & 1.195 & 1.194 & 1.194 & 1.195 & 1.193 & 1.186 & 1.195 \\
\hline LB & 20.796 & 21.195 & 19.971 & 21.438 & 19.861 & 20.288 & 20.631 & 33.222 & 29.055 & 30.176 & 31.262 & 30.403 & 32.697 & 31.269 \\
\hline
\end{tabular}

Diagnostics: Log Likelihood (LL), Bayes Information Criterion (BIC), mean $\left(\overline{\hat{\varepsilon}}_{i}\right)$, standard devation (SD) and Ljung-Box statistic with respect to 20 lags (LB) of ACD residuals. 
Table 11: P-values of the different types of diagnostic tests (see Table 2) for the estimates based on Disney trade durations and price durations (see Table 10).

\begin{tabular}{|c|c|c|c|c|c|c|c|c|c|c|c|c|c|c|}
\hline & \multicolumn{7}{|c|}{ Trade durations } & \multicolumn{6}{|c|}{ Price durations } & \multirow[b]{2}{*}{$(7)$} \\
\hline & (1) & (2) & $(3)$ & $(4)$ & $(5)$ & (6) & (7) & (1) & $(2)$ & $(3)$ & $(4)$ & (5) & (6) & \\
\hline \multicolumn{15}{|c|}{ LM tests } \\
\hline$L M_{1}$ & 0.859 & 0.841 & 0.996 & 0.973 & 0.998 & 1.000 & 1.000 & 0.000 & 0.000 & 0.994 & 0.743 & 1.000 & 1.000 & 1.000 \\
\hline$L M_{2}$ & 0.937 & .925 & 0.996 & 0.994 & 0.999 & 1.000 & 1.000 & 0.000 & 0.000 & 0.105 & 0.007 & 0.296 & 0.208 & 0.539 \\
\hline$L M_{3}$ & 0.841 & 0.809 & 0.994 & 0.993 & 0.997 & 1.000 & 1.000 & .000 & 0.000 & 0.968 & 0.622 & 0.996 & 1.000 & 0.999 \\
\hline $\mathrm{LM}_{4}$ & 0.937 & 909 & 0.996 & 0.999 & 0.998 & 1.000 & 1.000 & .000 & 0.000 & 0.118 & 0.008 & 0.387 & 0.298 & 0.633 \\
\hline$L M_{5}$ & 0.673 & 632 & 0.979 & 0.982 & 0.993 & 0.998 & 0.997 & 0.000 & 0.000 & 0.994 & 0.804 & 0.999 & 1.000 & 1.000 \\
\hline$L M_{6}$ & 0.817 & 0.781 & 0.981 & 0.969 & 0.994 & 0.993 & 0.985 & 0.000 & 0.000 & 0.228 & 0.034 & 0.289 & 0.303 & 0.767 \\
\hline$L M_{7}$ & 804 & 692 & 0.973 & .936 & 0.990 & 1.000 & 0.978 & .000 & 0.000 & 0.190 & 0.008 & 0.385 & 0.226 & 0.844 \\
\hline$L M_{8}$ & 0.877 & 0.850 & 0.972 & 0.985 & 0.984 & 1.000 & 0.998 & 0.000 & 0.000 & 0.119 & 0.001 & 0.473 & 0.266 & 0.773 \\
\hline \multicolumn{15}{|c|}{ CM tests } \\
\hline$C M_{1}^{1}$ & .197 & 240 & 0.441 & .443 & 0.116 & 0.559 & 0.439 & 0.000 & 0.000 & 0.682 & 0.350 & 0.621 & 0.296 & 0.279 \\
\hline$C M_{2}^{1}$ & 0.080 & 110 & 0.379 & 0.267 & 0.082 & 0.462 & 0.362 & .000 & 0.000 & 0.611 & 0.350 & 0.601 & 0.308 & 0.178 \\
\hline$C M_{3}^{1}$ & 0.267 & 0.285 & 0.543 & 0.370 & 0.253 & 0.507 & 0.404 & 0.000 & 0.000 & 0.048 & 0.002 & 0.050 & 0.003 & 0.033 \\
\hline$C M_{4}^{1}$ & 0.148 & 0.172 & 0.467 & 0.263 & 0.193 & 0.444 & 0.332 & 0.000 & 0.000 & 0.101 & 0.011 & 0.125 & 0.019 & 0.053 \\
\hline$C M_{5}^{1}$ & 0.558 & 0.530 & 0.783 & 0.404 & 0.672 & 0.910 & 0.661 & 0.000 & 0.000 & 0.690 & 0.388 & 0.649 & 0.342 & 0.216 \\
\hline$C M_{6}^{1}$ & 0.593 & 0.576 & 0.815 & 0.509 & 0.739 & 0.900 & 0.794 & .000 & 0.000 & 0.754 & 0.440 & 0.801 & 0.121 & 0.213 \\
\hline$C M_{7}^{1}$ & 0.708 & 0.731 & 0.851 & 0.548 & 0.850 & 0.974 & 0.666 & .000 & 0.000 & 0.015 & 0.001 & 0.015 & 0.000 & 0.005 \\
\hline$C M_{8}^{1}$ & 0.719 & 0.747 & 0.904 & 0.577 & 0.911 & 0.950 & 0.739 & 0.000 & 0.000 & 0.053 & 0.011 & 0.065 & 0.001 & 0.021 \\
\hline$C M_{9}^{1}$ & 0.598 & 0.553 & 0.788 & 0.617 & 0.642 & 0.856 & 0.678 & 0.000 & 0.000 & 0.485 & 0.206 & 0.458 & 0.417 & 0.155 \\
\hline$C M_{10}^{1}$ & 0.653 & 0.617 & 0.818 & 0.613 & 0.722 & 0.863 & 0.816 & 0.000 & 0.000 & 0.536 & 0.211 & 0.585 & 0.299 & 0.157 \\
\hline$C M_{11}^{1}$ & 0.740 & 0.741 & 0.839 & 0.730 & 0.772 & 0.951 & 0.563 & 0.000 & 0.000 & 0.016 & 0.001 & 0.017 & 0.000 & 0.010 \\
\hline$C M_{12}^{1}$ & 0.770 & 0.780 & 0.893 & 0.690 & 0.851 & 0.930 & 0.646 & 0.000 & 0.000 & 0.063 & 0.005 & 0.077 & 0.001 & 0.039 \\
\hline$C M_{1}^{2}$ & 0.604 & 0.585 & 0.816 & 0.635 & 0.755 & 0.745 & 0.778 & 0.000 & 0.000 & 0.621 & 0.158 & 0.545 & 0.224 & 0.153 \\
\hline$C M_{2}^{2}$ & 0.773 & 0.777 & 0.922 & 0.769 & 0.893 & 0.891 & 0.936 & 0.000 & 0.000 & 0.463 & 0.048 & 0.576 & 0.036 & 0.113 \\
\hline$C M_{3}^{2}$ & 0.850 & 0.852 & 0.928 & 0.798 & 0.908 & 0.839 & 0.713 & 0.000 & 0.000 & 0.024 & 0.001 & 0.016 & 0.000 & 0.010 \\
\hline$C M_{4}^{2}$ & 0.920 & 0.937 & 0.981 & 0.879 & 0.973 & 0.940 & 0.845 & 0.000 & 0.000 & 0.048 & 0.004 & 0.059 & 0.001 & 0.026 \\
\hline$C M_{5}^{2}$ & 0.676 & 0.490 & 0.752 & 0.580 & 0.786 & 0.773 & 0.693 & 0.004 & 0.004 & 0.009 & 0.004 & 0.012 & 0.002 & 0.014 \\
\hline$C M_{6}^{2}$ & 0.859 & 0.818 & 0.795 & 0.815 & 0.817 & 0.833 & 0.739 & 0.000 & 0.000 & 0.041 & 0.011 & 0.039 & 0.004 & 0.078 \\
\hline$C M_{7}^{2}$ & 0.594 & 0.414 & 0.783 & 0.510 & 0.801 & 0.776 & 0.713 & 0.001 & 0.001 & 0.002 & 0.001 & 0.003 & 0.000 & 0.000 \\
\hline$C M_{8}^{2}$ & 0.594 & 0.414 & 0.783 & 0.510 & 0.801 & 0.776 & 0.713 & 0.001 & 0.001 & 0.002 & 0.001 & 0.003 & 0.000 & 0.000 \\
\hline$C M_{9}^{2}$ & 0.591 & 0.440 & 0.845 & 0.708 & 0.801 & 0.959 & 0.640 & 0.000 & 0.000 & 0.055 & 0.002 & 0.036 & 0.005 & 0.074 \\
\hline$C M_{10}^{2}$ & 0.668 & 0.506 & 0.887 & 0.775 & 0.825 & 0.974 & 0.768 & 0.000 & 0.000 & 0.167 & 0.042 & 0.090 & 0.033 & 0.434 \\
\hline$C M_{11}^{2}$ & 0.677 & 0.635 & 0.753 & 0.826 & 0.665 & 0.904 & 0.733 & 0.000 & 0.000 & 0.025 & 0.000 & 0.018 & 0.011 & 0.031 \\
\hline$C M_{12}^{2}$ & 0.729 & 0.686 & 0.747 & 0.826 & 0.664 & 0.905 & 0.718 & 0.000 & 0.000 & 0.085 & 0.003 & 0.025 & 0.084 & 0.133 \\
\hline \multicolumn{15}{|c|}{ F-tests against nonlinearites in the news response } \\
\hline$N L_{1}$ & 0.448 & 0.276 & 0.692 & 0.551 & 0.587 & 0.710 & $\overline{0.572}$ & 0.000 & 0.000 & 0.900 & 0.240 & 0.856 & 0.663 & 0.990 \\
\hline$N L_{2}$ & 0.787 & 0.627 & 0.945 & 0.862 & 0.905 & 0.949 & 0.895 & 0.000 & 0.000 & 0.219 & 0.034 & 0.246 & 0.041 & 0.635 \\
\hline$N L_{3}$ & 0.600 & 0.559 & 0.728 & 0.691 & 0.640 & 0.701 & 0.849 & 0.000 & 0.000 & 0.149 & 0.001 & 0.173 & 0.045 & 0.504 \\
\hline$N L_{4}$ & 0.883 & 0.856 & 0.923 & 0.921 & 0.877 & 0.910 & 0.932 & 0.000 & 0.000 & 0.251 & 0.004 & 0.227 & 0.107 & 0.583 \\
\hline \multicolumn{15}{|c|}{ ICM tests } \\
\hline$I C M_{1}$ & 0.040 & 0.020 & 0.020 & 0.070 & 0.040 & 0.060 & 0.040 & 0.030 & 0.010 & 0.650 & 0.220 & 0.630 & 0.220 & 0.400 \\
\hline$I C M_{2}$ & 0.010 & 0.010 & 0.050 & 0.000 & 0.010 & 0.010 & 0.020 & 0.020 & 0.000 & 0.710 & 0.190 & 0.690 & 0.150 & 0.460 \\
\hline$I C M_{3}$ & 0.040 & 0.030 & 0.020 & 0.020 & 0.040 & 0.040 & 0.000 & 0.040 & 0.000 & 0.560 & 0.130 & 0.530 & 0.200 & 0.470 \\
\hline
\end{tabular}


Table 12: QML estimates of various types of ACD models for GE trade durations and $\$ 0.05$ price durations. Data extracted from the TAQ database available from the NYSE, sample period from 03/19/01 to 03/30/01 for trade durations and $01 / 02 / 01$ to $05 / 30 / 01$ for price durations. Standard errors based on OPG estimates (in brackets). NPACD model estimated based on the category bounds $(0.1,0.2,0.5,1.0,1.5,2.0,3.0)$ with $\bar{\varepsilon}_{0}=1.0$. The threshold values for the three-regime TACD model are fixed exogenously to 0.25 and 1.50 . The estimates of the TACD model are shown in the same rows as those of the SPACD model.

\begin{tabular}{|c|c|c|c|c|c|c|c|c|c|c|c|c|c|c|}
\hline & $(1)$ & $(2)$ & (3) & (4) & (5) & (6) & (7) & $(1)$ & $(2)$ & (3) & (4) & (5) & (6) & (7) \\
\hline & $\mathrm{ACD}$ & LACD & BACD I & EXACD & AGACD & SPACD & TACD & $\mathrm{ACD}$ & LACD & BACD I & EXACD & AGACD & SPACD & D TACD \\
\hline \multirow[t]{2}{*}{$\omega$} & 0.036 & -0.038 & -0.003 & -0.037 & 0.005 & -0.007 & 0.060 & 0.009 & -0.030 & -0.093 & -0.034 & -0.016 & 0.012 & -0.002 \\
\hline & $(0.005)$ & $(0.003)$ & $(0.030)$ & $(0.003)$ & $(0.022)$ & $(0.007)$ & $(0.036)$ & $(0.002)$ & $(0.004)$ & $(0.017)$ & $(0.005)$ & $(0.003)$ & $(0.006)$ & $(0.006)$ \\
\hline \multirow[t]{2}{*}{$\alpha_{1}$} & -0.001 & -0.001 & $-0.000-$ & -0.014 & -0.091 & & -0.215 & 0.143 & 0.122 & 0.372 & 0.174 & -0.389 & & 0.788 \\
\hline & $(0.006)$ & $(0.006)$ & $(0.006)$ & $(0.010)$ & $(0.037)$ & & $(0.131)$ & $(0.009)$ & $(0.007)$ & $(0.049)$ & $(0.011)$ & $(0.062)$ & & $(0.158)$ \\
\hline \multirow[t]{2}{*}{$\alpha_{2}$} & 0.043 & 0.038 & 0.042 & 0.056 & 0.162 & & 0.051 & -0.094 & -0.092 & -0.269 & -0.128 & 0.325 & & -0.620 \\
\hline & $(0.006)$ & $(0.006)$ & $(0.033)$ & $(0.010)$ & $(0.069)$ & & $(0.096)$ & $(0.007)$ & $(0.006)$ & $(0.036)$ & $(0.010)$ & $(0.054)$ & & $(0.160)$ \\
\hline \multirow[t]{2}{*}{$\beta_{1}$} & 0.922 & 0.965 & 0.964 & 0.966 & 0.907 & 0.962 & 0.942 & 1.227 & 1.532 & 1.434 & 1.433 & 1.408 & 1.391 & 1.066 \\
\hline & (0.007) & $(0.005)$ & $(0.005)$ & $(0.005)$ & $(0.044)$ & $(0.005)$ & $(0.033)$ & $(0.073)$ & $(0.057)$ & $(0.063)$ & $(0.067)$ & $(0.066)$ & $(0.068)$ & $(0.093)$ \\
\hline \multirow[t]{2}{*}{$\beta_{2}$} & & & & & & & & $-0.284-$ & & -0.442 & -0.442 & -0.417 & -0.400 & -0.137 \\
\hline & & & & & & & & $(0.066)$ & $(0.056)$ & $(0.062)$ & $(0.066)$ & $(0.065)$ & $(0.067)$ & $(0.081)$ \\
\hline \multirow[t]{2}{*}{$\delta_{1}$} & & & 0.705 & & 2.078 & & & & & $-^{*}$ & & $-^{*}$ & & \\
\hline & & & $(0.558)$ & & (1.223) & & & & & $-^{*}$ & & $-^{*}$ & & \\
\hline \multirow[t]{2}{*}{$\delta_{2}$} & & & 0.758 & & 0.702 & & & & & 0.407 & & 1.000 & & \\
\hline & & & $(0.107)$ & & (0.098) & & & & & $(0.051)$ & & - & & \\
\hline \multirow[t]{2}{*}{$b$} & & & & & 0.430 & & & & & & & 0.355 & & \\
\hline & & & & & $(0.052)$ & & & & & & & $(0.032)$ & & \\
\hline \multirow[t]{2}{*}{$c_{1}$} & & & & 0.023 & 1.000 & & & & & & -0.100 & -1.235 & & \\
\hline & & & & $(0.014)$ & - & & & & & & $(0.014)$ & $(0.043)$ & & \\
\hline \multirow[t]{2}{*}{$c_{2}$} & & & & -0.031 & 0.573 & & & & & & 0.082 & -1.179 & & \\
\hline & & & & $(0.014)$ & $(0.398)$ & & & & & & $(0.014)$ & $(0.041)$ & & \\
\hline$\nu_{1}$ & & & & & $\begin{array}{c}0.084 \\
(0.038)\end{array}$ & & & & & & & $0.000^{* *}$ & & \\
\hline$\nu_{2}$ & & & & & -0.046 & & & & & & & $0.000^{* *}$ & & \\
\hline \multirow{2}{*}{$\alpha_{1}^{+}\left(\omega^{2}\right)$} & & & & & $(0.002)$ & 0.039 & 0.031 & & & & & & 0.020 & 0.005 \\
\hline & & & & & & $(0.014)$ & $(0.012)$ & & & & & & $(0.012)$ & $(0.007)$ \\
\hline \multirow[t]{2}{*}{$\alpha_{2}^{+}\left(\alpha_{1}^{2}\right)$} & & & & & & -0.021 & -0.001 & & & & & & 0.051 & 0.147 \\
\hline & & & & & & $(0.027)$ & $(0.018)$ & & & & & & $(0.024)$ & )$(0.026)$ \\
\hline \multirow[t]{2}{*}{$\alpha_{3}^{+}\left(\alpha_{2}^{2}\right)$} & & & & & & -0.014 & 0.051 & & & & & & 0.002 & -0.097 \\
\hline & & & & & & $(0.023)$ & $(0.018)$ & & & & & & $(0.020)$ & $(0.027)$ \\
\hline \multirow{2}{*}{$\alpha_{4}^{+}\left(\beta_{1}^{2}\right)$} & & & & & & -0.008 & 0.918 & & & & & & 0.018 & 1.253 \\
\hline & & & & & & $(0.012)$ & $(0.013)$ & & & & & & (0.009) & $(0.086)$ \\
\hline \multirow[t]{2}{*}{$\alpha_{1}^{-}\left(\beta_{2}^{2}\right)$} & & & & & & -0.030 & & & & & & & 0.211 & -0.299 \\
\hline & & & & & & $(0.019)$ & & & & & & & $(0.017)$ & $(0.077)$ \\
\hline$\alpha_{2}^{-}\left(\omega^{3}\right)$ & & & & & & -0.048 & 0.080 & & & & & & 0.223 & 0.032 \\
\hline & & & & & & $(0.030)$ & $(0.028)$ & & & & & & $(0.029)$ & (0.019) \\
\hline$\alpha_{3}^{-}\left(\alpha_{1}^{3}\right)$ & & & & & & -0.227 & -0.014 & & & & & & 0.120 & 0.083 \\
\hline & & & & & & $(0.096)$ & $(0.006)$ & & & & & & $(0.072)$ & $(0.016)$ \\
\hline$\alpha_{4}^{-}\left(\alpha_{2}^{3}\right)$ & & & & & & 0.636 & 0.041 & & & & & & 0.512 & -0.044 \\
\hline & & & & & & (1.290) & $(0.006)$ & & & & & & $(0.181)$ & $(0.017)$ \\
\hline$a^{+}\left(\beta_{1}^{3}\right)$ & & & & & & 0.097 & 0.922 & & & & & & -0.377 & 1.328 \\
\hline & & & & & & $(0.031)$ & $(0.028)$ & & & & & & $(0.028)$ & $(0.097)$ \\
\hline$a^{-}\left(\beta_{2}^{3}\right)$ & & & & & & 0.006 & & & & & & & -0.039 & -0.392 \\
\hline & & & & & & $(0.005)$ & & & & & & & $(0.006)$ & $(0.088)$ \\
\hline$\overline{\text { Obs }}$ & 25101 & 25101 & 25101 & 25101 & 25101 & 25101 & 25101 & & 08 & 16008 & 08 & 16008 & 16008 & 16008 \\
\hline LL & -24813 & -24815 & -24811 & -24813 & -24800 & -24801 & -24804 & -14705 & -14706 & -14670 & -14674 & -14660 & -14662 & $2-14665$ \\
\hline $\mathrm{BIC}$ & -24834 & -24836 & -24841 & -24843 & -24851 & -24862 & -24865 & -14729 & -14730 & -14699 & -14708 & -14699 & -14725 & $5-14737$ \\
\hline$\overline{\hat{\varepsilon}}_{i}$ & 1.001 & 1.000 & 0.999 & 1.000 & 1.000 & 1.001 & 1.000 & 1.002 & 1.001 & 1.000 & 1.001 & 1.000 & 1.000 & 1.000 \\
\hline SD & 1.007 & 1.007 & 1.006 & 1.007 & 1.006 & 1.005 & 1.006 & 1.060 & 1.057 & 1.059 & 1.059 & 1.056 & 1.057 & 1.059 \\
\hline LB & 38.695 & 37.281 & 36.779 & 37.039 & 40.831 & 36.380 & 39.267 & 33.643 & 23.238 & 35.240 & 29.765 & 32.469 & 33.801 & 36.461 \\
\hline
\end{tabular}

Diagnostics: Log Likelihood (LL), Bayes Information Criterion (BIC), mean $\left(\overline{\hat{\varepsilon}}_{i}\right)$, standard devation (SD) and Ljung-Box statistic with respect to 20 lags (LB) of ACD residuals.

${ }^{*}$ : Estimation based on logarithmic model. ${ }^{* *}$ : Parameters set to zero. 
Table 13: P-values of the different types of diagnostic tests (see Table 2) for the estimates based on GE trade durations and price durations (see Table 12).

\begin{tabular}{|c|c|c|c|c|c|c|c|c|c|c|c|c|c|c|}
\hline & \multicolumn{7}{|c|}{ Trade durations } & \multicolumn{6}{|c|}{ Price durations } & \multirow[b]{2}{*}{ (7) } \\
\hline & (1) & (2) & $(3)$ & $(4)$ & $(5)$ & (6) & (7) & (1) & $(2)$ & $(3)$ & $(4)$ & (5) & (6) & \\
\hline \multicolumn{15}{|c|}{ LM tests } \\
\hline$L M_{1}$ & 0.457 & 0.823 & 0.877 & 0.846 & 0.992 & 1.000 & 0.793 & 0.000 & 0.000 & 0.851 & 0.921 & 0.985 & 1.000 & 1.000 \\
\hline$L M_{2}$ & 0.142 & 0.506 & 0.755 & 0.897 & 0.857 & 0.999 & 0.588 & 0.000 & 0.000 & 0.539 & 0.679 & 0.282 & 0.870 & 0.986 \\
\hline$L M_{3}$ & 0.973 & 0.948 & 0.681 & 1.000 & 0.998 & 1.000 & 0.997 & .000 & 0.000 & 0.588 & 0.414 & 0.618 & 0.993 & .999 \\
\hline $\mathrm{LM}_{4}$ & 0.529 & 0.330 & 0.145 & 0.950 & 0.800 & 0.958 & 0.941 & .000 & 0.000 & 0.028 & 0.022 & 0.009 & 0.201 & 0.288 \\
\hline$L M_{5}$ & 0.283 & 0.398 & 0.326 & 0.338 & 0.974 & 0.941 & 0.542 & 0.000 & 0.000 & 0.147 & 0.067 & 0.404 & 0.982 & 0.887 \\
\hline$L M_{6}$ & 0.080 & 0.104 & 0.137 & 0.124 & 0.831 & 0.602 & 0.336 & .000 & 0.000 & 0.022 & 0.006 & 0.010 & 0.318 & 0.073 \\
\hline$L M_{7}$ & 404 & 455 & 0.520 & 0.745 & 0.634 & 0.849 & 0.726 & .000 & 0.000 & 0.438 & 0.117 & 0.481 & 0.176 & 0.986 \\
\hline$L M_{8}$ & 0.808 & 0.812 & 0.882 & 0.996 & 0.880 & 928 & 0.765 & 0.000 & 0.000 & 0.068 & 0.028 & 0.020 & 0.291 & 0.203 \\
\hline \multicolumn{15}{|c|}{ CM tests } \\
\hline$C M_{1}^{1}$ & 0.036 & 0.063 & 0.079 & 0.000 & 0.403 & 0.147 & 0.026 & 0.000 & 0.000 & 0.221 & 0.053 & 0.011 & 0.022 & 0.044 \\
\hline$C M_{2}^{1}$ & 0.021 & .037 & 0.047 & 0.000 & 0.281 & 0.122 & 0.014 & .001 & 0.000 & 0.379 & 0.101 & 0.024 & 0.051 & 0.083 \\
\hline$C M_{3}^{1}$ & 0.005 & 0.008 & 0.034 & 0.000 & 0.118 & 0.035 & 0.009 & .001 & 0.000 & 0.145 & 0.069 & 0.022 & 0.029 & 0.018 \\
\hline$C M_{4}^{1}$ & 0.007 & 0.011 & 0.045 & 0.000 & 0.140 & 0.052 & 0.007 & 0.001 & 0.000 & 0.254 & 0.083 & 0.017 & 0.039 & 0.040 \\
\hline$C M_{5}^{1}$ & 0.010 & 0.021 & 0.008 & 0.000 & 0.182 & 0.052 & 0.002 & 0.000 & 0.000 & 0.003 & 0.003 & 0.009 & 0.154 & 0.003 \\
\hline$C M_{6}^{1}$ & 0.007 & 0.011 & 0.004 & 0.000 & 0.167 & 0.024 & 0.002 & .000 & 0.000 & 0.014 & 0.022 & 0.014 & 0.246 & 0.016 \\
\hline$C M_{7}^{1}$ & 0.001 & 0.002 & 0.003 & 0.000 & 0.056 & 0.007 & 0.001 & .000 & 0.000 & 0.000 & 0.000 & 0.000 & 0.011 & 0.000 \\
\hline$C M_{8}^{1}$ & 0.001 & 0.002 & 0.004 & 0.000 & 0.054 & 0.006 & 0.001 & .000 & 0.000 & 0.004 & 0.002 & 0.000 & 0.024 & 0.000 \\
\hline$C M_{9}^{1}$ & 0.014 & 0.027 & 0.014 & 0.000 & 0.251 & 0.089 & 0.005 & 0.000 & 0.000 & 0.000 & 0.000 & 0.001 & 0.023 & 0.000 \\
\hline$C M_{10}^{1}$ & 0.007 & 0.011 & 0.005 & 0.000 & 0.214 & 0.056 & 0.003 & 0.000 & 0.000 & 0.001 & 0.001 & 0.001 & 0.036 & 0.000 \\
\hline$C M_{11}^{1}$ & 0.001 & 0.002 & 0.003 & 0.000 & 0.060 & 0.008 & 0.001 & 0.000 & 0.000 & 0.000 & 0.000 & 0.000 & 0.000 & 0.000 \\
\hline$C M_{12}^{1}$ & 0.001 & 0.002 & 0.004 & 0.000 & 0.054 & 0.008 & 0.001 & 0.000 & 0.000 & 0.000 & 0.000 & 0.000 & 0.001 & 0.000 \\
\hline$C M_{1}^{2}$ & 0.033 & 0.070 & 0.021 & 0.000 & 0.328 & 0.089 & 0.006 & 0.000 & 0.000 & 0.000 & 0.001 & 0.003 & 0.039 & 0.000 \\
\hline$C M_{2}^{2}$ & 0.022 & 0.040 & 0.015 & 0.000 & 0.354 & 0.068 & 0.007 & 0.000 & 0.000 & 0.003 & 0.002 & 0.005 & 0.057 & 0.001 \\
\hline$C M_{3}^{2}$ & 0.008 & 0.015 & 0.016 & 0.000 & 0.199 & 0.024 & 0.006 & 0.000 & 0.000 & 0.000 & 0.000 & 0.000 & 0.001 & 0.000 \\
\hline$C M_{4}^{2}$ & 0.008 & 0.015 & 0.024 & 0.000 & 0.202 & 0.033 & 0.006 & 0.000 & 0.000 & 0.000 & 0.000 & 0.000 & 0.003 & 0.000 \\
\hline$C M_{5}^{2}$ & 0.000 & 0.000 & 0.000 & 0.000 & 0.001 & 0.001 & 0.000 & 0.111 & 0.000 & 0.025 & 0.034 & 0.022 & 0.010 & 0.004 \\
\hline$C M_{6}^{2}$ & 0.000 & 0.000 & 0.000 & 0.000 & 0.003 & 0.000 & 0.000 & 0.018 & 0.000 & 0.151 & 0.118 & 0.112 & 0.073 & 0.028 \\
\hline$C M_{7}^{2}$ & 0.003 & 0.000 & 0.001 & 0.000 & 0.001 & 0.006 & 0.001 & 0.002 & 0.000 & 0.000 & 0.001 & 0.001 & 0.000 & 0.001 \\
\hline$C M_{8}^{2}$ & 0.003 & 0.000 & 0.001 & 0.000 & 0.001 & 0.006 & 0.001 & 0.002 & 0.000 & 0.000 & 0.001 & 0.001 & 0.000 & 0.001 \\
\hline$C M_{9}^{2}$ & 0.122 & 0.134 & 0.052 & 0.000 & 0.087 & 0.175 & 0.039 & 0.000 & 0.000 & 0.000 & 0.000 & 0.000 & 0.000 & 0.002 \\
\hline$C M_{10}^{2}$ & 0.063 & 0.072 & 0.025 & 0.000 & 0.072 & 0.045 & 0.013 & 0.000 & 0.000 & 0.000 & 0.000 & 0.000 & 0.000 & 0.000 \\
\hline$C M_{11}^{2}$ & 0.461 & 0.488 & 0.369 & 0.000 & 0.226 & 0.288 & 0.026 & 0.000 & 0.000 & 0.000 & 0.000 & 0.000 & 0.000 & 0.000 \\
\hline$C M_{12}^{2}$ & 0.313 & 0.358 & 0.237 & 0.000 & 0.193 & 0.106 & 0.012 & 0.000 & 0.000 & 0.000 & 0.000 & 0.000 & 0.000 & 0.000 \\
\hline \multicolumn{15}{|c|}{ F-tests against nonlinearites in the news response } \\
\hline$N L_{1}$ & 0.332 & 0.274 & 0.285 & 0.013 & 0.219 & 0.540 & 0.237 & 0.000 & 0.000 & 0.048 & 0.001 & 0.396 & 0.061 & 0.806 \\
\hline$N L_{2}$ & 0.163 & 0.217 & 0.211 & 0.000 & 0.177 & 0.408 & 0.215 & 0.000 & 0.000 & 0.016 & 0.000 & 0.079 & 0.001 & 0.175 \\
\hline$N L_{3}$ & 0.574 & 0.567 & 0.525 & 0.020 & 0.419 & 0.676 & 0.364 & 0.000 & 0.000 & 0.002 & 0.000 & 0.012 & 0.031 & 0.019 \\
\hline$N L_{4}$ & 0.406 & 0.432 & 0.462 & 0.000 & 0.320 & 0.396 & 0.265 & 0.000 & 0.000 & 0.001 & 0.000 & 0.001 & 0.002 & 0.001 \\
\hline \multicolumn{15}{|c|}{ ICM tests } \\
\hline$I C M_{1}$ & 0.340 & 0.400 & 0.360 & 0.850 & 0.810 & 0.270 & 0.560 & 0.950 & 0.000 & 0.870 & 0.970 & 0.940 & 0.990 & 0.930 \\
\hline$I C M_{2}$ & 0.260 & 0.390 & 0.310 & 0.770 & 0.650 & 0.360 & 0.500 & 0.900 & 0.030 & 0.870 & 0.920 & 0.910 & 0.940 & 0.930 \\
\hline$I C M_{3}$ & 0.300 & 0.300 & 0.240 & 0.750 & 0.590 & 0.290 & 0.520 & 0.900 & 0.000 & 0.700 & 0.940 & 0.920 & 0.920 & 0.780 \\
\hline
\end{tabular}




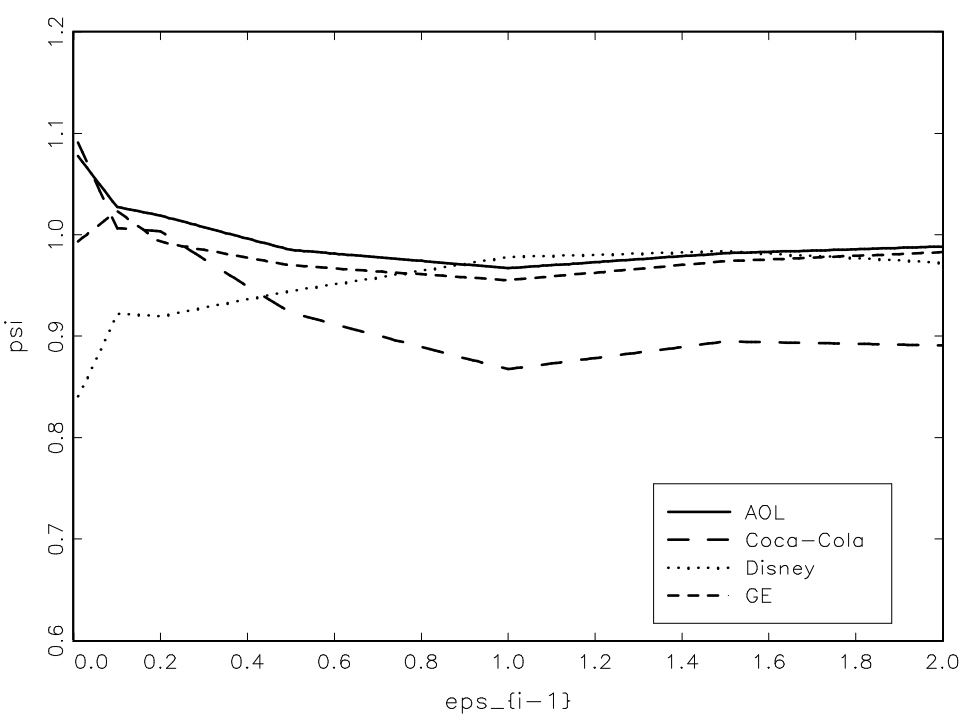

Figure 2: Estimated news impact curves for trade durations of the AOL, Coca-Cola, Disney and GE stock.

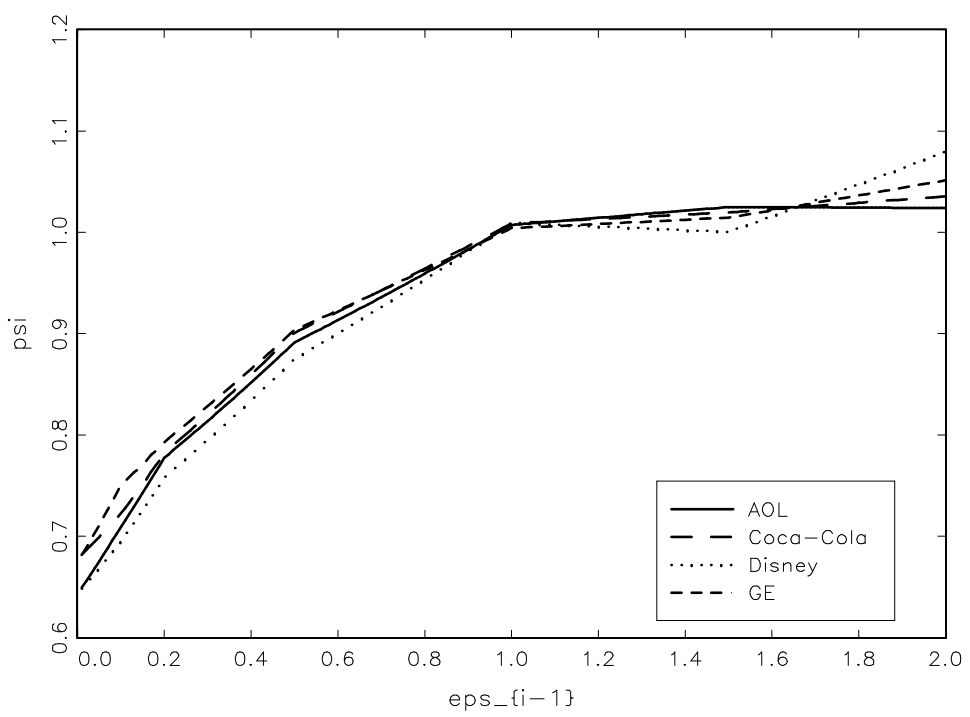

Figure 3: Estimated news impact curves for price durations of the AOL, Coca-Cola, Disney and GE stock. 